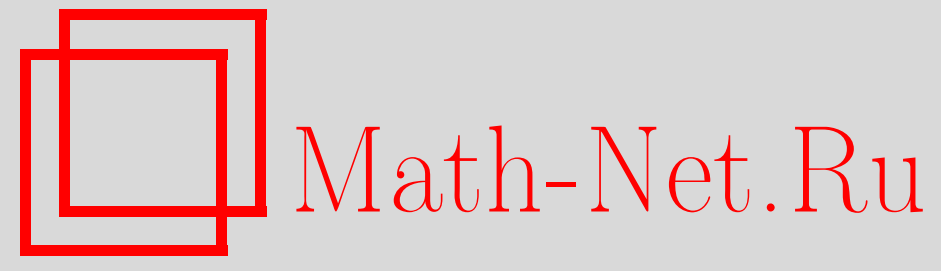

I. Jana, K. Saha, A. B. Soshnikov, Fluctuations of linear eigenvalue statistics of random band matrices, Теория вероятн. и ее примен., 2015, том 60, выпуск 3, 553-592

DOI: https://doi.org/10.4213/tvp4637

Использование Общероссийского математического портала Math-Net.Ru подразумевает, что вы прочитали и согласны с пользовательским соглашением http://www . mathnet.ru/rus/agreement

Параметры загрузки:

IP: 3.91 .87 .62

26 апреля 2023 г., $16: 17: 39$

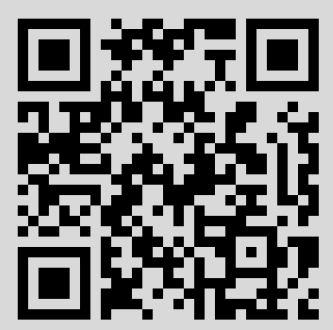




\section{FLUCTUATIONS OF LINEAR EIGENVALUE STATISTICS OF RANDOM BAND MATRICES}

В этой статье мы изучаем флуктуацию линейных статистик собственных значений случайных ленточных матриц $M_{n}=$ $W_{n} / \sqrt{b_{n}}$, где $W_{n}$ есть $n \times n$ эрмитова случайная матрица с ленточной длинной $b_{n}$, то есть только диагональные и первые $b_{n}$ внедиагональные матричные элементы не равны нулю. Мы изучаем линейные статистики $\mathscr{N}(\phi)=\sum_{i=1}^{n} \phi\left(\lambda_{i}\right)$, где $\lambda_{i}-$ собственные значения $M_{n}$ и $\phi$ достаточно гладкая тест-функция. Мы доказываем, что $\sqrt{b_{n} / n}[\mathscr{N}(\phi)-\mathbb{E} \mathscr{N}(\phi)] \stackrel{d}{\rightarrow} N(0, V(\phi))$ при $b_{n} \gg \sqrt{n}$, где выражение для $V(\phi)$ даётся в Теореме 1.

Ключевые слова и фразы: случайная ленточная матрица, центральная предельная теорема, нормальное распределение, линейные статистики собственных значений, полукруговой закон Вигнера, случайные матрицы Вигнера.

1. Introduction. Random matrix theory was developed from several different sources in the early 20th century. It is used as an important mathematical tool in various fields namely, mathematics, physics, wireless communication engineering, etc. One of the earliest example of a random matrix appeared in the study of sample covariance estimation was done by John Wishart [21]. In the early 1950s, Wigner introduced random matrix ensemble to study the energy spectra of heavy atoms undergoing slow nuclear reactions.

Random matrices are also used to model wireless channels. A random matrix model of CDMA networks can be found in [17], [18].

A special kind of random matrix ensemble is a random band matrix. In 1955, Wigner studied the matrices $H$ of the form $H=K+V$, where $K$ is an $n \times n$ diagonal matrix consisting of $\ldots,-2,-1,0,1,2, \ldots$, and $V$ is

*Department of Mathematics, University of California, Davis, CA; e-mail: ijana@math.ucdavis.edu

** Department of Mathematics, Indian Institute of Technology, Bombay, Mumbai, India; ksaha@math.iitb.ac.in

*** Department of Mathematics, University of California, Davis, CA; soshniko@math.ucdavis.edu

1) Research supported by INSPIRE Fellowship, Dept. of Science and Technology, Govt. of India and VIMSS fellowship. 
an $n \times n$ symmetric sign matrix having nonvanishing elements only up to a distance $b_{n}$ from the main diagonal. Such a matrix $H$ was called as bordered matrix [19], [20].

Another treatment of random band matrix was done by G. Casati et al. [4], [3] in the context of quantum chaos. They studied $n \times n$ symmetric random band matrices of bandwidth $b_{n}$, where $b_{n}$ grows with $n$. In 1992, Molchanov et al. proved the semicircle law for random band matrices [12]. In 1991, Fyodorov and Mirlin proved that $b_{n}^{2} / n$ is a crucial parameter for random band matrices [8], [11]. Numerical simulations show that the local eigenvalue statistics changes from Poisson to GOE or GUE as $b_{n}$ changes from $b_{n} \ll \sqrt{n}$ to $b_{n} \gg \sqrt{n}$. Recently, Li and Soshnikov [9] proved the central limit theorem (CLT) for linear statistics of eigenvalues of band random matrices when the bandwidth $b_{n}$ satisfies $\sqrt{n} \ll b_{n} \ll n$. In this article we write $\alpha_{n} \ll \beta_{n}$ if $\alpha_{n} / \beta_{n} \rightarrow 0$ as $n \rightarrow \infty$, and we write $\alpha_{n}=O\left(\beta_{n}\right)$ if $\left|\alpha_{n} / \beta_{n}\right| \leqslant C$ for all $n$ for some constant $C>0$.

In this article, we deal with the CLT for the eigenvalue statistics of band random matrices. We take the approach of M. Shcherbina in [15] to establish the CLT for band matrices with bandwidth $b_{n}$ where $b_{n} \rightarrow \infty$ as $n \rightarrow \infty$. We give an alternative proof of Li and Soshnikov [9] result on CLT of band matrices when $\sqrt{n} \ll b_{n} \ll n$. We have given some simulation results in Section 5, which ensure that the CLT for band matrices will also hold if $\sqrt{n} / b_{n} \nrightarrow 0$ and $b_{n} \rightarrow \infty$.

Now we define our model. Let us define the (circular) distance function $d_{n}: \mathbb{N} \times \mathbb{N} \rightarrow \mathbb{N}$ as

$$
d_{n}(j, k):=\min \{|j-k|, n-|j-k|\},
$$

and the index sets $I_{n}, I_{n}^{+} \subset \mathbb{N} \times \mathbb{N}, I_{1} \subset \mathbb{N}$ as

$$
\begin{gathered}
I_{n}:=\left\{(j, k): d_{n}(j, k) \leqslant b_{n}\right\}, \quad I_{n}^{+}=\left\{(j, k):(j, k) \in I_{n}, j \leqslant k\right\}, \\
I_{1}=\left\{1<j \leqslant n:(1, j) \in I_{n}\right\},
\end{gathered}
$$

where $\left\{b_{n}\right\}$ is a sequence of positive integers such that $b_{n} \rightarrow \infty$ as $n \rightarrow \infty$.

Define a real symmetric random band matrix $M=\left(m_{j k}\right)_{n \times n}$ of bandwidth $b_{n}$ as

$$
m_{j k}=m_{k j}= \begin{cases}b_{n}^{-1 / 2} w_{j k} & \text { if } d_{n}(j, k) \leqslant b_{n} \\ 0 & \text { otherwise }\end{cases}
$$

where $\left\{w_{i i}\right\}$ and $\left\{w_{j k}\right\}_{j \neq k,(j, k) \in I_{n}^{+}}$are two sets of independent identically distributed (i.i.d.) real random variables with

$$
\mathbb{E}\left[w_{j k}\right]=0, \quad \mathbb{E}\left[w_{j k}^{2}\right]=\left\{\begin{array}{cc}
1 & \text { if } j \neq k \\
\sigma^{2} & \text { if } j=k .
\end{array}\right.
$$


Here $\left\{w_{j k}\right\}$ may depend on $n$, but we suppress it when there is no confusion. Let $\lambda_{1} \leqslant \lambda_{2} \leqslant \cdots \leqslant \lambda_{n}$ be the eigenvalues of the random band matrix $M$. Define the linear eigenvalue statistic of the eigenvalues of $M$ as

$$
\mathscr{N}_{n}(\phi)=\sum_{i=1}^{n} \phi\left(\lambda_{i}\right)
$$

and the normalized eigenvalue statistic of the matrix $M$ as

$$
\mathscr{M}_{n}(\phi)=\sqrt{\frac{b_{n}}{n}} \mathscr{N}_{n}(\phi)
$$

where $\phi$ is a test function.

\section{Main results.}

Theorem 1. Let $M$ be a real symmetric random band matrix as defined in (2), and $b_{n}$ be a sequence of integers satisfying $\sqrt{n} \ll b_{n} \ll n$. Assume the following:

(i) $w_{j k}$ satisfies the Poincaré inequality with constant $m>0$ not depending on $j, k, n$, i.e., for any continuously differentiable function $f$,

$$
\mathbf{D}\left(f\left(w_{j k}\right)\right) \leqslant \frac{1}{m} \mathbb{E}\left[\left|f^{\prime}\left(w_{j k}\right)\right|^{2}\right] .
$$

(ii) $\mathbb{E}\left[w_{j k}^{4}\right]=\mu_{4}$ for all $j \neq k$ and $d_{n}(j, k) \leqslant b_{n}$.

(iii) $\phi: \mathbb{R} \rightarrow \mathbb{R}$ is a test function in the Sobolev space $H^{s}$, i.e., $\|\phi\|_{s}<\infty$, where

$$
\|\phi\|_{s}^{2}=\int_{\mathbb{R}}(1+2|t|)^{2 s}|\widehat{\phi}(t)|^{2} d t, \quad \widehat{\phi}(t)=\frac{1}{\sqrt{2 \pi}} \int_{\mathbb{R}} e^{-i t \lambda} \phi(\lambda) d \lambda,
$$

and $s>5 / 2$.

Then the centred normalized eigenvalue statistic $\mathscr{M}^{\circ}(\phi)=\mathscr{M}_{n}(\phi)-$ $\mathbb{E}\left[\mathscr{M}_{n}(\phi)\right]$ converges in distribution to the Gaussian random variable with mean zero and variance given by

$$
\begin{aligned}
V(\phi)= & \frac{\kappa_{4}}{16 \pi^{2}}\left(\int_{-2 \sqrt{2}}^{2 \sqrt{2}} \frac{4-\mu^{2}}{\sqrt{8-\mu^{2}}} \phi(\mu) d \mu\right)^{2}+\frac{\sigma^{2}}{16 \pi^{2}}\left(\int_{-2 \sqrt{2}}^{2 \sqrt{2}} \frac{\mu \phi(\mu)}{\sqrt{8-\mu^{2}}} d \mu\right)^{2} \\
& +\int_{-2 \sqrt{2}}^{2 \sqrt{2}} \int_{-2 \sqrt{2}}^{2 \sqrt{2}} \sqrt{\left(8-x^{2}\right)\left(8-y^{2}\right)} F(x, y) \\
& \times \int_{-2 \sqrt{2}}^{2 \sqrt{2}} \int_{-2 \sqrt{2}}^{2 \sqrt{2}} \frac{\mu_{1} \phi\left(\mu_{1}\right)}{\left(x-\mu_{1}\right) \sqrt{8-\mu_{1}^{2}}} \frac{\mu_{2} \phi\left(\mu_{2}\right)}{\left(x-\mu_{2}\right)^{2} \sqrt{8-\mu_{2}^{2}}} d \mu_{1} d \mu_{2} d x d y,
\end{aligned}
$$

where for $x \neq y$

$F(x, y)=2 \int_{-\infty}^{\infty} \frac{s^{3} \sin s-s \sin ^{3} s}{2\left(s^{2}-\sin ^{2} s\right)^{2}-\left(s^{3} \sin s+s \sin ^{3} s\right) x y+s^{2} \sin ^{2} s\left(x^{2}+y^{2}\right)} d s$,

and $\kappa_{4}$ is the fourth cumulant of the off-diagonal entries, i.e., $\kappa_{4}=\mu_{4}-3$. 
3. Proof of Theorem 1. We will follow the approach taken by M. Shcherbina in [15] for full (Wigner) matrix. This approach is based on two main ideas. The first ingredient is stated in the following proposition which gives a bound on the variance of linear eigenvalue statistics with a sufficiently smooth test function in term of the variance of the trace of the resolvent of a random matrix. For a proof of this result see [15], [13]. In what follows, we denote $X^{\circ}=X-\mathbb{E}[X]$ for any random variable $X$.

Proposition 1. Let $M$ be an $n \times n$ real symmetric random matrix and $\mathscr{N}_{n}(\phi)$ be a linear eigenvalue statistic of its eigenvalue as in (4). Then for any $s>0$ we have

$$
\mathbf{D}\left[\mathscr{N}_{n}(\phi)\right] \leqslant C_{s}\|\phi\|_{s}^{2} \int_{0}^{\infty} d y e^{-y} y^{2 s-1} \int_{-\infty}^{\infty} \mathbf{D}[\operatorname{Tr}(G(x+i y))] d x,
$$

where $C_{s}$ is a constant depends only on $s$, and $G(z)=(M-z I)^{-1}$ is the resolvent of the matrix $M$.

The second ingredient of this approach is to use the martingale difference technique to provide a good bound on $\mathbf{D}\left(\gamma_{n}\right)$, where $\gamma_{n}$ is the trace of the resolvent of a matrix. The following proposition gives that bound.

Proposition 2. Consider the symmetric band matrix $M$ defined in (2) and assume (3) is satisfied. Then for some $C>0$ not depending on $z, n$ we have

$$
\mathbf{D}\left\{\gamma_{n}\right\} \leqslant \frac{C n}{b_{n}}\left(y^{-2}+y^{-4}\right)\left(\max \left\{y,|x|-\frac{2}{y}\right\}\right)^{-2},
$$

where $\gamma_{n}=\operatorname{Tr}(M-z I)^{-1}=\operatorname{Tr}(G)$ and $z=x+i y, y>0$.

We prove this result in the appendix section. Now we outline the proof of Theorem 1.

P r o o f o f T h e o r e m 1. By Lévy's continuity theorem, it suffices to show that if

$$
Z_{n}(x)=\mathbb{E}\left[e_{n}(x)\right], \quad e_{n}(x)=e^{i x \mathscr{M}_{n}^{\circ}(\phi)},
$$

then for each $x \in \mathbb{R}$

$$
\lim _{n \rightarrow \infty} Z_{n}(x)=\exp \left[-\frac{x^{2} V(\phi)}{2}\right]
$$

with $V(\phi)$ as in Theorem 1. For any test function $\phi \in H^{s}$, define

$$
\phi_{\eta}=P_{\eta} * \phi,
$$

where $P_{\eta}$ is the Poisson kernel given by

$$
P_{\eta}(x)=\frac{\eta}{\pi\left(x^{2}+\eta^{2}\right)}
$$


We know that $\phi_{\eta}$ approximates $\phi$ in the $H^{s}$ norm, i.e.,

$$
\lim _{\eta \rightarrow 0}\left\|\phi-\phi_{\eta}\right\|_{s} \rightarrow 0
$$

For the moment, we denote the characteristic function defined in (7), by $Z_{n}(\phi)$ (to make its dependence on $\phi$ clear). Then we have

$$
\lim _{n \rightarrow \infty} Z_{n}(\phi)=\lim _{\eta \downarrow 0} \lim _{n \rightarrow \infty}\left(Z_{n}(\phi)-Z_{n}\left(\phi_{\eta}\right)\right)+\lim _{\eta \downarrow 0} \lim _{n \rightarrow \infty} Z_{n}\left(\phi_{\eta}\right) .
$$

Now using the Proposition 1 and (8), we shall show that

$$
\lim _{\eta \downarrow 0} \lim _{n \rightarrow \infty}\left(Z_{n}(\phi)-Z_{n}\left(\phi_{\eta}\right)\right)=0
$$

and then $\lim _{n \rightarrow \infty} Z_{n}(\phi)=\lim _{\eta \downarrow} \lim _{n \rightarrow \infty} Z_{n}\left(\phi_{\eta}\right)$. Hence it suffices to find the limit of

$$
Z_{\eta, n}:=Z_{n}\left(\phi_{\eta}\right)=\mathbb{E}\left[e_{\eta, n}(x)\right]
$$

with

$$
e_{\eta, n}(x)=\exp \left[i x \mathscr{M}_{n}^{\circ}\left(\phi_{\eta}\right)\right]
$$

as $n \rightarrow \infty$ and $\eta \downarrow 0$ uniformly in $n$. Proofs of (9) and (10) are given in the next two subsections and that will complete the proof of this theorem.

3.1. Proof of equation (9). First observe that

$$
\begin{aligned}
\left|Z_{n}(\phi)-Z_{n}\left(\phi_{\eta}\right)\right|^{2} & \leqslant 2|x|^{2} \mathbf{D}\left[\mathscr{M}_{n}(\phi)-\mathscr{M}_{n}\left(\phi_{\eta}\right)\right] \\
& \leqslant 2|x|^{2} \frac{b_{n}}{n} \mathbf{D}\left[\mathscr{N}_{n}(\phi)-\mathscr{N}_{n}\left(\phi_{\eta}\right)\right] .
\end{aligned}
$$

Now, in view of Proposition 1 , to bound $\mathbf{D}\left[\mathscr{N}_{n}(\phi)-\mathscr{N}_{n}\left(\phi_{\eta}\right)\right]$ we need to estimate

$$
\int_{-\infty}^{\infty} \mathbf{D}\left(\gamma_{n}(x+i y)\right) d x
$$

where $\gamma_{n}(x+i y)=\operatorname{Tr}(G(x+i y))$ and $G(z)=(M-z I)^{-1}$. We estimate that for $y>0$

$$
\begin{aligned}
\int_{-\infty}^{\infty}\left(\max \left\{y,|x|-\frac{2}{y}\right\}\right)^{-2} d x & \leqslant \int_{|| x|-2 / y|<y} \frac{1}{y^{2}} d x \\
& +\int_{|| x|-2 / y| \geqslant y}(x-2 / y)^{-2} d x \\
\leqslant & \frac{10}{y}+10 y .
\end{aligned}
$$


Using the above estimate and (6), we have

$$
\begin{gathered}
\int_{0}^{\infty} d y e^{-y} y^{2 s-1} \int_{-\infty}^{\infty} \mathbf{D}\left(\gamma_{n}\right) d x \leqslant \frac{C^{\prime}}{b_{n}} \int_{0}^{\infty} e^{-y} y^{2 s-1} 4 n\left(\frac{1}{y}+y\right)\left(\frac{1}{y^{2}}+\frac{1}{y^{4}}\right) d y \\
=C \frac{n}{b_{n}} \int_{0}^{\infty} e^{-y}\left(2 y^{2 s-3-1}+y^{2 s-1-1}+y^{2 s-5-1}\right) d y \\
=C \frac{n}{b_{n}}(\Gamma(2 s-3)+\Gamma(2 s-1)+\Gamma(2 s-5)) .
\end{gathered}
$$

If we take

$$
s=\frac{5}{2}+\varepsilon, \quad \varepsilon>0,
$$

then $\Gamma(2 s-3)=\Gamma(2+2 \varepsilon), \Gamma(2 s-1)=\Gamma(4+2 \varepsilon)$, and $\Gamma(2 s-5)=\Gamma(2 \varepsilon)$. By Proposition 1, and (12), we have

$$
\mathbf{D}\left(\mathscr{N}_{n}(\phi)-\mathscr{N}_{n}\left(\phi_{\eta}\right)\right) \leqslant C(\varepsilon) \frac{n}{b_{n}}\left\|\phi-\phi_{\eta}\right\|_{s} .
$$

Using the above estimate and (11), we have

$$
\begin{aligned}
\left|Z_{n}(\phi)-Z_{n}\left(\phi_{\eta}\right)\right|^{2} & \leqslant 2|x|^{2} \frac{b_{n}}{n} C(\varepsilon) \frac{n}{b_{n}}\left\|\phi-\phi_{\eta}\right\|_{s} \\
& =2 C(\varepsilon)|x|^{2}\left\|\phi-\phi_{\eta}\right\|_{s} \rightarrow 0 \quad \text { as } \eta \rightarrow 0 .
\end{aligned}
$$

The last limit follows from the equation (8). This completes the proof of (9).

3.2. Finding the limit of the characteristic function (10). We will be using the Lemma 1 and Lemma 2 from appendix in the proof of (10). Denote the averaging with respect to $\left\{w_{1 i} ; 1 \leqslant i \leqslant n\right\}$ by $\mathbb{E}_{1}$.

$\mathrm{P} r$ o o f of (10). Using the dominated convergence theorem we have

$$
\begin{aligned}
\frac{d}{d x} Z_{n}\left(\phi_{\eta}\right) & =\frac{d}{d x} \mathbb{E}\left[e_{\eta, n}(x)\right]=\frac{d}{d x} \mathbb{E}\left[\exp \left(i x \sqrt{\frac{b_{n}}{n}} \mathscr{N}_{n}^{\circ}\left(\phi_{\eta}\right)\right)\right] \\
& =\mathbb{E}\left[i \sqrt{\frac{b_{n}}{n}} \mathscr{N}_{n}^{\circ}\left(\phi_{\eta}\right) e_{\eta, n}(x)\right] .
\end{aligned}
$$

Since by construction $\phi_{\eta}=P_{\eta} * \phi$, we have

$$
\mathscr{N}_{n}^{\circ}\left(\phi_{\eta}\right)=\frac{1}{\pi} \int_{-\infty}^{\infty} \phi(\mu) \operatorname{Im} \gamma_{n}^{\circ}\left(z_{\mu}\right) d \mu, \quad \text { where } z_{\mu}=\mu+i \eta .
$$

Hereinafter, we use the finiteness of $\int_{\mathbb{R}}|\phi(\mu)| d \mu$ for $\phi \in H^{s}, s>1 / 2$, when changing the order of integration. For notational convenience, from now on 
we will denote $e_{\eta, n}(x)$ by $e(x)$. Therefore,

$$
\begin{aligned}
\frac{d}{d x} Z_{n}\left(\phi_{\eta}\right) & =\mathbb{E}\left[i \sqrt{\frac{b_{n}}{n}} e(x) \frac{1}{\pi} \int_{-\infty}^{\infty} \phi(\mu) \operatorname{Im} \gamma_{n}^{\circ}\left(z_{\mu}\right) d \mu\right] \\
& =\frac{1}{2 \pi} \sqrt{\frac{b_{n}}{n}} \int_{-\infty}^{\infty} \phi(\mu) \mathbb{E}\left[e(x) \operatorname{Tr}\left(G^{\circ}\left(z_{\mu}\right)-G^{\circ}\left(\bar{z}_{\mu}\right)\right)\right] d \mu \\
& =\frac{1}{2 \pi} \sqrt{\frac{b_{n}}{n}} \int_{-\infty}^{\infty} \phi(\mu)\left(Y_{n}\left(z_{\mu}, x\right)-Y_{n}\left(\bar{z}_{\mu}, x\right)\right) d \mu,
\end{aligned}
$$

where

$$
\begin{aligned}
Y_{n}(z, x) & =\mathbb{E}\left[e(x) \operatorname{Tr}\left(G^{\circ}(z)\right)\right]=\mathbb{E}\left[e^{\circ}(x) \operatorname{Tr}(G(z))\right]=n \mathbb{E}\left[G_{11}(z) e^{\circ}(x)\right] \\
& =-n \mathbb{E}\left[\left(A^{-1}\right)^{\circ} e_{1}(x)\right]-n \mathbb{E}\left[\left(A^{-1}\right)^{\circ}\left(e(x)-e_{1}(x)\right)\right], \quad(13) \\
e_{1}(x) & =\exp \left[i x \sqrt{\frac{b_{n}}{n}}\left(\mathscr{N}_{n-1}\left(\phi_{\eta}\right)\right)^{\circ}\right], \\
\left(\mathscr{N}_{n-1}\left(\phi_{\eta}\right)\right)^{\circ} & =\frac{1}{\pi} \int_{-\infty}^{\infty} \phi(\mu) \operatorname{Im}\left(\gamma_{n-1}(z)\right)^{\circ} d \mu, \\
\gamma_{n-1}(z) & =\operatorname{Tr} G^{(1)}(z), \\
A(z) & =z-\frac{1}{\sqrt{b_{n}}} w_{11}+\left\langle G^{(1)} m^{(1)}, m^{(1)}\right\rangle, \\
m^{(1)} & =\frac{1}{\sqrt{b_{n}}}\left(w_{12}, w_{13}, \ldots, w_{1 n}\right)^{T}, \\
G^{(1)}(z) & =\left(G_{i j}^{(1)}(z)\right)_{i, j=2}^{n}=\left(M^{(1)}-z I\right)^{-1},
\end{aligned}
$$

and $M^{(1)}$ is the main bottom $(n-1) \times(n-1)$ minor of $M$. In the above notation $\langle\cdot, \cdot\rangle$ represents the inner product of two complex vectors, i.e., $\langle x, y\rangle=\bar{y}^{T} x$ for $x, y \in \mathbb{C}^{n-1}$. The equation (13) follows from the Schur complement lemma, which says that

$$
G_{11}(z)=\frac{1}{w_{11} / \sqrt{b_{n}}-z-\left\langle G^{(1)} m^{(1)}, m^{(1)}\right\rangle}=-\frac{1}{A(z)} .
$$

Now we rewrite

$$
\begin{aligned}
\sqrt{\frac{b_{n}}{n}} Y_{n}(z, x) & =-\sqrt{n b_{n}} \mathbb{E}\left[\left(A^{-1}\right)^{\circ} e_{1}(x)\right]-\sqrt{n b_{n}} \mathbb{E}\left[\left(A^{-1}\right)^{\circ}\left(e(x)-e_{1}(x)\right)\right] \\
& =: T_{1}+T_{2} .
\end{aligned}
$$

Using Taylor expansion we have

$$
A^{-1}=\frac{1}{\mathbb{E}[A]}-\frac{A^{\circ}}{(\mathbb{E}[A])^{2}}+\frac{\left(A^{\circ}\right)^{2}}{(\mathbb{E}[A])^{3}}-\frac{\left(A^{\circ}\right)^{3}}{(\mathbb{E}[A])^{4}}+\frac{\left(A^{\circ}\right)^{4}}{A(\mathbb{E}[A])^{4}} .
$$


Therefore, we can estimate

$$
\begin{aligned}
& T_{1}=-\sqrt{n b_{n}} \mathbb{E}\left[\left(A^{-1}\right)^{\circ} e_{1}(x)\right] \\
&=-\sqrt{n b_{n}} \mathbb{E}\left[\left(A^{-1}\right) e_{1}^{\circ}(x)\right] \\
&=-\sqrt{n b_{n}} \mathbb{E}\left[\left(\frac{1}{\mathbb{E}[A]}-\frac{A^{\circ}}{(\mathbb{E}[A])^{2}}+\frac{\left(A^{\circ}\right)^{2}}{(\mathbb{E}[A])^{3}}\right.\right. \\
&\left.\left.\quad-\frac{\left(A^{\circ}\right)^{3}}{(\mathbb{E}[A])^{4}}+\frac{\left(A^{\circ}\right)^{4}}{A(\mathbb{E}[A])^{4}}\right) e_{1}^{\circ}(x)\right] \\
&= \sqrt{n b_{n}} \mathbb{E}\left[\left(\frac{A^{\circ}}{(\mathbb{E}[A])^{2}}-\frac{\left(A^{\circ}\right)^{2}}{(\mathbb{E}[A])^{3}}\right) e_{1}^{\circ}(x)\right] \\
&+ \sqrt{n b_{n}} \mathbb{E}\left[\left(\frac{\left(A^{\circ}\right)^{3}}{(\mathbb{E}[A])^{4}}-\frac{\left(A^{\circ}\right)^{4}}{A(\mathbb{E}[A])^{4}}\right) e_{1}^{\circ}(x)\right] .
\end{aligned}
$$

Now we shall estimate each term individually. First of all, since $M$ is a real symmetric matrix we have

$$
\|G(z)\| \leqslant \frac{1}{|\operatorname{Im} z|},
$$

and, in particular, $1 /|A| \leqslant 1 /|\operatorname{Im} z|$. It can also be checked that $1 /|\mathbb{E}[A]| \leqslant$ $1 /|\operatorname{Im} z|$. Hereinafter $\|X\|$ is the spectral norm of a matrix $X$. Using the above equation (21) and the estimates (37), (39), we have

$$
\begin{aligned}
\left|\sqrt{n b_{n}} \mathbb{E}\left[\frac{\left(A^{\circ}\right)^{4}}{A(\mathbb{E}[A])^{4}} e_{1}^{\circ}(x)\right]\right| & \leqslant \frac{\sqrt{n b_{n}}}{|\operatorname{Im} z|^{5}} \mathbb{E}\left[\left|\left(A^{\circ}\right)^{4}\right|\right] \\
& =\frac{\sqrt{n b_{n}}}{|\operatorname{Im} z|^{5}} O\left(b_{n}^{-2}\right)=O\left(\sqrt{\frac{n}{b_{n}^{3}}}\right) \rightarrow 0, \\
\left|\sqrt{n b_{n}} \mathbb{E}\left[\frac{\left(A^{\circ}\right)^{3}}{(\mathbb{E}[A])^{4}} e_{1}^{\circ}(x)\right]\right| & \leqslant \frac{\sqrt{n b_{n}}}{|\operatorname{Im} z|^{4}} \mathbb{E}\left[\left|\left(A^{\circ}\right)^{3}\right|\right] \\
& =\frac{\sqrt{n b_{n}}}{|\operatorname{Im} z|^{4}} O\left(b_{n}^{-3 / 2}\right)=O\left(\sqrt{\frac{n}{b_{n}^{2}}}\right) \rightarrow 0, \\
\left|\sqrt{n b_{n}} \mathbb{E}\left[\frac{\left(A^{\circ}\right)^{2}}{(\mathbb{E}[A])^{3}} e_{1}^{\circ}(x)\right]\right| & \leqslant \frac{\sqrt{n b_{n}}}{|\operatorname{Im} z|^{3}}\left|\mathbb{E}\left[e_{1}^{\circ}(x) \mathbb{E}_{1}\left[\left(A^{\circ}\right)^{2}\right]\right]\right| \\
& \leqslant C \sqrt{\frac{n}{b_{n}}}\left|\mathbb{E}\left[e_{1}^{\circ}(x)\left(b_{n} \mathbb{E}_{1}\left(A^{\circ}\right)^{2}\right)\right]\right| \\
& \leqslant C \sqrt{\frac{n}{b_{n}}}\left[\mathbf{D}\left(e_{1}^{\circ}(x)\right)\right]^{1 / 2}\left[\mathbf{D}\left(b_{n} \mathbb{E}_{1}\left(A^{\circ}\right)^{2}\right)\right]^{1 / 2} \\
& \leqslant C \sqrt{\frac{n}{b_{n}}} O\left(b_{n}^{-1 / 2}\right) \\
& =O\left(\sqrt{\frac{n}{b_{n}^{2}}}\right) \rightarrow 0 \quad \text { as } n \rightarrow \infty .
\end{aligned}
$$


Therefore, we have

$$
\begin{aligned}
T_{1} & =\frac{\sqrt{n b_{n}}}{(\mathbb{E}[A])^{2}} \mathbb{E}\left[A^{\circ} e_{1}^{\circ}(x)\right]+O\left(\sqrt{\frac{n}{b_{n}^{2}}}\right) \\
& =\frac{\sqrt{n b_{n}}}{(\mathbb{E}[A])^{2}} \mathbb{E}\left[e_{1}^{\circ}(x) \mathbb{E}_{1}\left(A^{\circ}\right)\right]+O\left(\sqrt{\frac{n}{b_{n}^{2}}}\right) .
\end{aligned}
$$

Now

$$
A^{\circ}=-\frac{1}{\sqrt{b_{n}}} w_{11}+\frac{1}{b_{n}} \sum_{\substack{i \neq j \\ i, j \in I_{1}}} G_{i j}^{(1)} w_{1 i} w_{1 j}+\frac{1}{b_{n}} \sum_{i \in I_{1}}\left(G_{i i}^{(1)} w_{1 i}^{2}-\mathbb{E}\left[G_{i i}^{(1)}\right]\right),
$$

where $I_{1}=\left\{1<j \leqslant n:(1, j) \in I_{n}\right\}$.

Therefore,

$$
\mathbb{E}_{1}\left[A^{\circ}(z)\right]=\frac{1}{b_{n}} \sum_{i \in I_{1}}\left(G_{i i}^{(1)}-\mathbb{E}\left[G_{i i}^{(1)}\right]\right)
$$

and hence

$$
\begin{aligned}
T_{1} & =\frac{\sqrt{n b_{n}}}{(\mathbb{E}[A])^{2}} \mathbb{E}\left[e_{1}^{\circ}(x) \mathbb{E}_{1}\left(A^{\circ}\right)\right]+O\left(\sqrt{\frac{n}{b_{n}^{2}}}\right) \\
& =\frac{\sqrt{n b_{n}}}{(\mathbb{E}[A])^{2}} \mathbb{E}\left[e_{1}^{\circ}(x) \frac{1}{b_{n}} \sum_{i \in I_{1}}\left(G_{i i}^{(1)}-\mathbb{E}\left[G_{i i}^{(1)}\right]\right)\right]+O\left(\sqrt{\frac{n}{b_{n}^{2}}}\right) \\
& =\frac{\sqrt{n b_{n}}}{(\mathbb{E}[A])^{2}} 2 \mathbb{E}\left[\left(G_{22}^{(1)}\right)^{\circ} e_{1}^{\circ}(x)\right]+O\left(\sqrt{\frac{n}{b_{n}^{2}}}\right) \\
& =\frac{\sqrt{n b_{n}}}{(\mathbb{E}[A])^{2}} \frac{2}{n} \mathbb{E}\left[\gamma_{n-1}^{\circ} e_{1}^{\circ}(x)\right]+O\left(\sqrt{\frac{n}{b_{n}^{2}}}\right) \\
& =\sqrt{\frac{b_{n}}{n}} \frac{2}{(\mathbb{E}[A])^{2}} \mathbb{E}\left[\gamma_{n-1}^{\circ} e_{1}(x)\right]+O\left(\sqrt{\frac{n}{b_{n}^{2}}}\right) .
\end{aligned}
$$

Hereinafter, all bounds (implicitly) depending on $z$ hold uniformly on the set $\{\mu+i \eta: \mu \in \mathbb{R}\}$ for any given $\eta>0$. Now

$$
\begin{aligned}
& \left|\mathbb{E}\left[\gamma_{n-1}^{\circ} e_{1}(x)\right]-\mathbb{E}\left[\gamma_{n}^{\circ} e(x)\right]\right| \\
& \quad=\left|\mathbb{E}\left[\gamma_{n-1}^{\circ} e_{1}(x)\right]-\mathbb{E}\left[\gamma_{n}^{\circ} e_{1}(x)\right]+\mathbb{E}\left[\gamma_{n}^{\circ} e_{1}(x)\right]-\mathbb{E}\left[\gamma_{n}^{\circ} e(x)\right]\right| \\
& \quad \leqslant\left(\mathbb{E}\left[\left|\gamma_{n-1}^{\circ}-\gamma_{n}^{\circ}\right|^{4}\right]\right)^{1 / 4}+\left|\mathbb{E}\left[\gamma_{n}^{\circ}\left(e_{1}(x)-e(x)\right)\right]\right| \\
& \quad=O\left(b_{n}^{-1 / 2}\right)+\left|\mathbb{E}\left[\gamma_{n}^{\circ}\left(e_{1}(x)-e(x)\right)\right]\right| .
\end{aligned}
$$


The last equality follows from (40). We estimate

$$
\begin{aligned}
e(x)-e_{1}(x)= & \exp \left[i x \sqrt{\frac{b_{n}}{n}} \mathscr{N}_{n}^{\circ}\left(\phi_{\eta}\right)\right]-\exp \left[i x \sqrt{\frac{b_{n}}{n}} \mathscr{N}_{n-1}^{\circ}\left(\phi_{\eta}\right)\right] \\
= & \left(\exp \left[i x \sqrt{\frac{b_{n}}{n}} \mathscr{N}_{n}^{\circ}\left(\phi_{\eta}\right)-i x \sqrt{\frac{b_{n}}{n}} \mathscr{N}_{n-1}^{\circ}\left(\phi_{\eta}\right)\right]-1\right) e_{1}(x) \\
= & i x \sqrt{\frac{b_{n}}{n}}\left(\mathscr{N}_{n}^{\circ}\left(\phi_{\eta}\right)-\mathscr{N}_{n-1}^{\circ}\left(\phi_{\eta}\right)\right) e_{1}(x) \\
& +\frac{b_{n}}{n} O\left(x^{2}\left(\mathscr{N}_{n}^{\circ}\left(\phi_{\eta}\right)-\mathscr{N}_{n-1}^{\circ}\left(\phi_{\eta}\right)\right)^{2} e_{1}(x)\right) \\
= & \frac{i x}{\pi} \sqrt{\frac{b_{n}}{n}} \int_{-\infty}^{\infty}\left[\phi(\mu) \operatorname{Im}\left(\gamma_{n}^{\circ}-\gamma_{n-1}^{\circ}\right) e_{1}(x)\right. \\
& \left.+\sqrt{\frac{b_{n}}{n}} \phi(\mu) O\left(\gamma_{n}^{\circ}-\gamma_{n-1}^{\circ}\right)^{2}\right] d \mu .
\end{aligned}
$$

Therefore,

$$
\begin{aligned}
\mathbb{E}\left[\gamma_{n}^{\circ}\left(e(x)-e_{1}(x)\right)\right]=\mathbb{E}\left[\frac{i x}{\pi} \sqrt{\frac{b_{n}}{n}} \int_{-\infty}^{\infty} \phi(\mu)\right. & {\left[\operatorname{Im}\left(\gamma_{n}^{\circ}-\gamma_{n-1}^{\circ}\right) e_{1}(x) \gamma_{n}^{\circ}\right.} \\
& \left.\left.+\sqrt{\frac{b_{n}}{n}} \gamma_{n}^{\circ} O\left(\gamma_{n}^{\circ}-\gamma_{n-1}^{\circ}\right)^{2}\right] d \mu\right] .
\end{aligned}
$$

Using estimates (6) and (40), we have

$$
\begin{aligned}
\left|\mathbb{E}\left[\operatorname{Im}\left(\gamma_{n}^{\circ}-\gamma_{n-1}^{\circ}\right) e_{1}(x) \gamma_{n}^{\circ}\right]\right| & \leqslant\left(\mathbb{E}\left[\left|\gamma_{n}^{\circ}\right|^{2}\right]\right)^{1 / 2}\left(\mathbb{E}\left[\left|e_{1}(x) \operatorname{Im}\left(\gamma_{n}^{\circ}-\gamma_{n-1}^{\circ}\right)\right|^{2}\right]\right)^{1 / 2} \\
& =O\left(\sqrt{\frac{n}{b_{n}}} \sqrt{\frac{1}{b_{n}}}\right) .
\end{aligned}
$$

Similarly,

$$
\mathbb{E}\left[\gamma_{n}^{\circ} O\left(\gamma_{n}^{\circ}-\gamma_{n-1}^{\circ}\right)^{2}\right]=O\left(\sqrt{\frac{n}{b_{n}}} \frac{1}{b_{n}}\right)
$$

Therefore,

$$
\left|\mathbb{E}\left[\gamma_{n-1}^{\circ} e_{1}(x)\right]-\mathbb{E}\left[\gamma_{n}^{\circ} e(x)\right]\right|=O\left(\frac{1}{\sqrt{b_{n}}}\right) .
$$

From the equation (22) and the above estimates we have

$$
T_{1}=\sqrt{\frac{b_{n}}{n}} \frac{2}{(\mathbb{E}[A])^{2}} \mathbb{E}\left[\gamma_{n-1}^{\circ} e_{1}(x)\right]+O\left(\frac{\sqrt{n}}{b_{n}}\right)
$$




$$
\begin{aligned}
& =\sqrt{\frac{b_{n}}{n}} \frac{2}{(\mathbb{E}[A])^{2}}\left[\mathbb{E}\left[\gamma_{n}^{\circ} e(x)\right]+O\left(\frac{1}{\sqrt{b_{n}}}\right)\right]+O\left(\frac{\sqrt{n}}{b_{n}}\right) \\
& =\sqrt{\frac{b_{n}}{n}} \frac{2}{(\mathbb{E}[A])^{2}} Y_{n}(z, x)+O\left(\frac{\sqrt{n}}{b_{n}}\right) .
\end{aligned}
$$

Now consider $T_{2}$. Using (23) and (30) we have

$$
\begin{aligned}
T_{2}= & -\sqrt{n b_{n}} \mathbb{E}\left[\left(A^{-1}\right)^{\circ}\left(e(x)-e_{1}(x)\right)\right] \\
= & -\frac{i x b_{n}}{\pi} \mathbb{E}\left[\left(A^{-1}\right)^{\circ} \int_{-\infty}^{\infty} \phi(\mu) \operatorname{Im}\left(\gamma_{n}^{\circ}-\gamma_{n-1}^{\circ}\right) e_{1}(x) d \mu\right] \\
& \left.-\frac{1}{\pi} \sqrt{\frac{b_{n}^{3}}{n}} \mathbb{E}\left[\left(A^{-1}\right)^{\circ} \int_{-\infty}^{\infty} \phi(\mu) O\left(\gamma_{n}^{\circ}-\gamma_{n-1}^{\circ}\right)^{2} d \mu\right]\right] \\
= & -\frac{i x b_{n}}{\pi} \int_{-\infty}^{\infty} \phi(\mu) \mathbb{E}\left[e_{1}(x)\left(A^{-1}\right)^{\circ} \operatorname{Im}\left(\gamma_{n}^{\circ}-\gamma_{n-1}^{\circ}\right)\right] d \mu+\sqrt{\frac{b_{n}^{3}}{n}} O\left(\frac{1}{b_{n}}\right) \\
= & -\frac{i x b_{n}}{\pi} \int_{-\infty}^{\infty} \phi(\mu) \mathbb{E}\left[e_{1}(x)\left(A^{-1}\right)^{\circ} \operatorname{Im}\left(\gamma_{n}^{\circ}-\gamma_{n-1}^{\circ}\right)\right] d \mu+O\left(\sqrt{\frac{b_{n}}{n}}\right) \\
= & -\frac{i x b_{n}}{\pi} \int_{-\infty}^{\infty} \phi(\mu) \mathbb{E}\left[e_{1}(x)\left(A^{-1}\right)^{\circ} \operatorname{Im}\left(\gamma_{n}-\gamma_{n-1}\right)^{\circ}\right] d \mu+O\left(\sqrt{\frac{b_{n}}{n}}\right) \\
= & \frac{i x b_{n}}{\pi} \int_{-\infty}^{\infty} \phi(\mu) \mathbb{E}\left[e_{1}(x)\left(A^{-1}\right)^{\circ}(z) \operatorname{Im}\left(\frac{1+B\left(z_{\mu}\right)}{A\left(z_{\mu}\right)}\right)^{\circ}\right] d \mu+O\left(\sqrt{\frac{b_{n}}{n}}\right) \\
= & T_{21}-T_{22}+O\left(\sqrt{\frac{b_{n}}{n}}\right),
\end{aligned}
$$

where $B(z)=\left\langle G^{(1)} G^{(1)} m^{(1)}, m^{(1)}\right\rangle$ and

$$
\begin{aligned}
& T_{21}=\frac{x b_{n}}{2 \pi} \int_{-\infty}^{\infty} \phi(\mu) \mathbb{E}\left[e_{1}(x)\left(A^{-1}\right)^{\circ}(z)\left(\frac{1+B\left(z_{\mu}\right)}{A\left(z_{\mu}\right)}\right)^{\circ}\right] d \mu, \\
& T_{22}=\frac{x b_{n}}{2 \pi} \int_{-\infty}^{\infty} \phi(\mu) \mathbb{E}\left[e_{1}(x)\left(A^{-1}\right)^{\circ}(z)\left(\frac{\overline{1+B\left(z_{\mu}\right)}}{\overline{A\left(z_{\mu}\right)}}\right)^{\circ}\right] d \mu .
\end{aligned}
$$

Using $\operatorname{Im}\left\langle G^{(1)} m^{(1)}, m^{(1)}\right\rangle=\operatorname{Im} z\left\langle G^{(1) *} G^{(1)} m^{(1)}, m^{(1)}\right\rangle$, it can be easily verified that

$$
\left|\frac{B(z)}{A(z)}\right| \leqslant \frac{1}{|\operatorname{Im} z|}, \quad \frac{1}{|\mathbb{E}[A(z)]|} \leqslant \frac{1}{|\operatorname{Im} z|}, \quad \text { and } \quad|\mathbb{E}[B(z)]| \leqslant \frac{2}{|\operatorname{Im} z|^{2}}
$$

Applying

$$
A^{-1}=\frac{1}{\mathbb{E}[A]}-\frac{A^{\circ}}{(\mathbb{E}[A])^{2}}+\frac{\left(A^{\circ}\right)^{2}}{A(\mathbb{E}[A])^{2}}
$$


to $A^{-1}(z), A^{-1}\left(z_{\mu}\right)$ and using $(37)$, we get

$$
\begin{aligned}
& b_{n} \mathbb{E}\left[e_{1}(x)\left(A^{-1}\right)^{\circ}(z)\left(\frac{1+B\left(z_{\mu}\right)}{A\left(z_{\mu}\right)}\right)^{\circ}\right] \\
& =b_{n} \mathbb{E}\left[e _ { 1 } ( x ) \left\{\frac { A ^ { \circ } ( z ) } { \mathbb { E } ^ { 2 } [ A ( z ) ] } \left(-\frac{B^{\circ}\left(z_{\mu}\right)}{\mathbb{E}\left[A\left(z_{\mu}\right)\right]}+\frac{\left(1+B\left(z_{\mu}\right)\right) A^{\circ}\left(z_{\mu}\right)}{\mathbb{E}^{2}\left[A\left(z_{\mu}\right)\right]}\right.\right.\right. \\
& \left.\left.\left.-\frac{\mathbb{E}\left[B\left(z_{\mu}\right) A^{\circ}\left(z_{\mu}\right)\right]}{\mathbb{E}^{2}\left[A\left(z_{\mu}\right)\right]}\right)\right\}\right]+O\left(b_{n}^{-1 / 2}\right) \\
& =b_{n} \mathbb{E}\left[e _ { 1 } ( x ) \left\{\frac { A ^ { \circ } ( z ) } { \mathbb { E } ^ { 2 } [ A ( z ) ] } \left(-\frac{B^{\circ}\left(z_{\mu}\right)}{\mathbb{E}\left[A\left(z_{\mu}\right)\right]}+\frac{\left(1+\mathbb{E}\left[B\left(z_{\mu}\right)\right]\right) A^{\circ}\left(z_{\mu}\right)}{\mathbb{E}^{2}\left[A\left(z_{\mu}\right)\right]}\right.\right.\right. \\
& \left.\left.\left.+\frac{\left(B^{\circ}\left(z_{\mu}\right) A^{\circ}\left(z_{\mu}\right)\right)^{\circ}}{\mathbb{E}^{2}\left[A\left(z_{\mu}\right)\right]}\right)\right\}\right]+O\left(b_{n}^{-1 / 2}\right) \\
& =\frac{\left(1+\mathbb{E}\left[B\left(z_{\mu}\right)\right]\right)}{\mathbb{E}^{2}[A(z)] \mathbb{E}^{2}\left[A\left(z_{\mu}\right)\right]} \mathbb{E}\left[e_{1}(x) b_{n} A^{\circ}(z) A^{\circ}\left(z_{\mu}\right)\right] \\
& -\frac{\mathbb{E}\left[e_{1}(x) b_{n} A^{\circ}(z) B^{\circ}\left(z_{\mu}\right)\right]}{\mathbb{E}^{2}[A(z)] \mathbb{E}\left[A\left(z_{\mu}\right)\right]}+O\left(b_{n}^{-1 / 2}\right) \\
& =\frac{\left(1+\mathbb{E}\left[B\left(z_{\mu}\right)\right]\right)}{\mathbb{E}^{2}[A(z)] \mathbb{E}^{2}\left[A\left(z_{\mu}\right)\right]} \mathbb{E}\left[e_{1}(x) \mathbb{E}_{1}\left(b_{n} A^{\circ}(z) A^{\circ}\left(z_{\mu}\right)\right)\right] \\
& -\frac{\mathbb{E}\left[e_{1}(x) \mathbb{E}_{1}\left[b_{n} A^{\circ}(z) B^{\circ}\left(z_{\mu}\right)\right]\right]}{\mathbb{E}^{2}[A(z)] \mathbb{E}\left[A\left(z_{\mu}\right)\right]}+O\left(b_{n}^{-1 / 2}\right) .
\end{aligned}
$$

Using (39), from the last expression we get

$$
\begin{aligned}
b_{n} \mathbb{E} & {\left[e_{1}(x)\left(A^{-1}\right)^{\circ}(z)\left(\frac{1+B\left(z_{\mu}\right)}{A\left(z_{\mu}\right)}\right)^{\circ}\right] } \\
= & \frac{\left(1+\mathbb{E} B\left(z_{\mu}\right)\right)}{\mathbb{E}^{2}[A(z)] \mathbb{E}^{2}\left[A\left(z_{\mu}\right)\right]} \mathbb{E}\left[e_{1}(x)\right] \mathbb{E}\left[b_{n} A^{\circ}(z) A^{\circ}\left(z_{\mu}\right)\right] \\
& -\frac{\mathbb{E}\left[e_{1}(x)\right] \mathbb{E}\left[b_{n} A^{\circ}(z) B^{\circ}\left(z_{\mu}\right)\right]}{\mathbb{E}^{2}[A(z)] \mathbb{E}\left[A\left(z_{\mu}\right)\right]}+O\left(b_{n}^{-1 / 2}\right) .
\end{aligned}
$$

Define

$$
\begin{aligned}
D_{n}\left(z, z_{\mu}\right)= & \frac{\left(1+\mathbb{E}\left[B\left(z_{\mu}\right)\right]\right) \mathbb{E}\left[b_{n} \mathbb{E}_{1}\left\{A^{\circ}(z) A^{\circ}\left(z_{\mu}\right)\right\}\right]}{\mathbb{E}^{2}[A(z)] \mathbb{E}^{2}\left[A\left(z_{\mu}\right)\right]} \\
& -\frac{\mathbb{E}\left[b_{n} \mathbb{E}_{1}\left\{A^{\circ}(z) B^{\circ}\left(z_{\mu}\right)\right\}\right]}{\mathbb{E}^{2}[A(z)] \mathbb{E}\left[A\left(z_{\mu}\right)\right]} .
\end{aligned}
$$

Also, using (23) and (40), we have

$$
\begin{aligned}
\mathbb{E}[e(x)]-\mathbb{E}\left[e_{1}(x)\right]=\mathbb{E}\left[\frac{i x}{\pi} \sqrt{\frac{b_{n}}{n}} \int_{-\infty}^{\infty} \phi(\mu) \operatorname{Im}\left(\gamma_{n}^{\circ}-\gamma_{n-1}^{\circ}\right) e_{1}(x) d \mu\right. \\
\left.\quad+\frac{b_{n}}{n} x^{2} \int_{-\infty}^{\infty} \phi(\mu) O\left(\gamma_{n}^{\circ}-\gamma_{n-1}^{\circ}\right)^{2} d \mu\right] \\
=O\left(n^{-1 / 2}\right)+O\left(n^{-1}\right) .
\end{aligned}
$$


Therefore,

$$
\mathbb{E}\left[e_{1}(x)\right]=Z_{n}\left(\phi_{\eta}\right)+O\left(n^{-1 / 2}\right) .
$$

Combining (18), (24), (26), and (27), we get

$$
\begin{aligned}
& \sqrt{\frac{b_{n}}{n}} Y_{n}(z, x)=T_{1}+T_{2} \\
&=\frac{2}{\mathbb{E}^{2}[A]} \sqrt{\frac{b_{n}}{n}} Y_{n}(z, x)+\frac{x}{2 \pi} \mathbb{E}\left[e_{1}(x)\right] \int_{-\infty}^{\infty}\left[D_{n}\left(z, z_{\mu}\right)-D_{n}\left(z, \bar{z}_{\mu}\right)\right] \phi(\mu) d \mu \\
& \quad+O\left(b_{n}^{-1 / 2}\right) \\
&=\frac{2}{\mathbb{E}^{2}[A]} \sqrt{\frac{b_{n}}{n}} Y_{n}(z, x)+\frac{x}{2 \pi} Z_{n}\left(\phi_{\eta}\right) \int_{-\infty}^{\infty}\left[D_{n}\left(z, z_{\mu}\right)-D_{n}\left(z, \bar{z}_{\mu}\right)\right] \phi(\mu) d \mu \\
& \quad+o(1) \\
& \approx 2 f^{2}(z) \tilde{Y}_{n}(z, x)+\frac{x}{2 \pi} Z_{n}\left(\phi_{\eta}\right) \int_{-\infty}^{\infty}\left[D_{n}\left(z, z_{\mu}\right)-D_{n}\left(z, \bar{z}_{\mu}\right)\right] \phi(\mu) d \mu+o(1),
\end{aligned}
$$

where $\tilde{Y}_{n}(z, x)=\sqrt{\frac{b_{n}}{n}} Y_{n}(z, x)$. Therefore,

$$
\tilde{Y}_{n}(z, x)=Z_{n}\left(\phi_{\eta}\right) \frac{x}{2 \pi} \int_{-\infty}^{\infty}\left(C_{n}\left(z, z_{\mu}\right)-C_{n}\left(z, \bar{z}_{\mu}\right)\right) \phi(\mu) d \mu+o(1)
$$

uniformly in $z$ with $\operatorname{Im} z=\eta$, where $C_{n}\left(z, z_{\mu}\right)=D_{n}\left(z, z_{\mu}\right) /\left(1-2 f^{2}(z)\right)$ and $f(z)$ is given in (41). Hence

$$
\begin{aligned}
\frac{d}{d x} & Z_{n}\left(\phi_{\eta}\right)=\frac{1}{2 \pi} \int_{-\infty}^{\infty} \phi(\mu)\left(\tilde{Y}_{n}\left(z_{\mu}, x\right)-\tilde{Y}_{n}\left(\bar{z}_{\mu}, x\right)\right) d \mu \\
= & \frac{1}{2 \pi} \int_{-\infty}^{\infty} \phi\left(\mu_{1}\right)\left[\frac{x}{2 \pi} Z_{n}\left(\phi_{\eta}\right) \int_{-\infty}^{\infty} \phi\left(\mu_{2}\right)\left(C_{n}\left(z_{\mu_{1}}, z_{\mu_{2}}\right)-C_{n}\left(z_{\mu_{1}}, \bar{z}_{\mu_{2}}\right)\right) d \mu_{2}\right. \\
& \left.\quad-\frac{x}{2 \pi} Z_{n}\left(\phi_{\eta}\right) \int_{-\infty}^{\infty} \phi\left(\mu_{2}\right)\left(C_{n}\left(\bar{z}_{\mu_{1}}, z_{\mu_{2}}\right)-C_{n}\left(\bar{z}_{\mu_{1}}, \bar{z}_{\mu_{2}}\right)\right) d \mu_{2}\right] d \mu_{1}+o(1) \\
= & -\frac{x}{4 \pi^{2}} Z_{n}\left(\phi_{\eta}\right) \int_{-\infty}^{\infty} \int_{-\infty}^{\infty} \phi\left(\mu_{1}\right) \phi\left(\mu_{2}\right)\left[C_{n}\left(z_{\mu_{1}}, \bar{z}_{\mu_{2}}\right)+C_{n}\left(\bar{z}_{\mu_{1}}, z_{\mu_{2}}\right)\right. \\
& \left.-C_{n}\left(z_{\mu_{1}}, z_{\mu_{2}}\right)-C_{n}\left(\bar{z}_{\mu_{1}}, \bar{z}_{\mu_{2}}\right)\right] d \mu_{2} d \mu_{1}+o(1) \\
= & -x Z_{n}\left(\phi_{\eta}\right) V_{n}(\phi, \eta)+o(1) .
\end{aligned}
$$

To find the limit of $V_{n}(\phi, \eta)$, we shall calculate limit of $\left[C_{n}\left(z_{\mu_{1}}, \bar{z}_{\mu_{2}}\right)+\right.$ $\left.C_{n}\left(\bar{z}_{\mu_{1}}, z_{\mu_{2}}\right)-C_{n}\left(z_{\mu_{1}}, z_{\mu_{2}}\right)-C_{n}\left(\bar{z}_{\mu_{1}}, \bar{z}_{\mu_{2}}\right)\right]$ as $n \rightarrow \infty$. Using (34) and (35),

$$
\begin{aligned}
D_{n}\left(z, z_{\mu}\right)= & \frac{\left(1+\mathbb{E}\left[B\left(z_{\mu}\right)\right]\right) \mathbb{E}\left[b_{n} \mathbb{E}_{1}\left\{A^{\circ}(z) A^{\circ}\left(z_{\mu}\right)\right\}\right]}{\mathbb{E}^{2}[A(z)] \mathbb{E}^{2}\left[A\left(z_{\mu}\right)\right]} \\
& -\frac{\mathbb{E}\left[b_{n} \mathbb{E}_{1}\left\{A^{\circ}(z) B^{\circ}\left(z_{\mu}\right)\right\}\right]}{\mathbb{E}^{2}[A(z)] \mathbb{E}\left[A\left(z_{\mu}\right)\right]}
\end{aligned}
$$




$$
\begin{aligned}
& =\frac{1+\mathbb{E}\left[B\left(z_{\mu}\right)\right]}{\mathbb{E}^{2}[A(z)] \mathbb{E}^{2}\left[A\left(z_{\mu}\right)\right]} \\
& \times \mathbb{E}\left[\frac{2}{b_{n}} \sum_{i, j \in I_{1}} G_{i j}^{(1)}(z) G_{i j}^{(1)}\left(z_{\mu}\right)+\sigma^{2}+\frac{\kappa_{4}}{b_{n}} \sum_{i \in I_{1}} G_{i i}^{(1)}(z) G_{i i}^{(1)}\left(z_{\mu}\right)\right. \\
& \left.\quad+\frac{1}{b_{n}} \widetilde{\gamma_{n-1}}(z) \widetilde{\gamma_{n-1}}\left(z_{\mu}\right)\right] \\
& -\frac{1}{\mathbb{E}^{2}[A(z)] \mathbb{E}\left[A\left(z_{\mu}\right)\right]} \frac{d}{d z_{\mu}} \mathbb{E}\left[\frac{2}{b_{n}} \sum_{i, j \in I_{1}} G_{i j}^{(1)}(z) G_{i j}^{(1)}\left(z_{\mu}\right)\right. \\
& \left.\quad+\sigma^{2}+\frac{\kappa_{4}}{b_{n}} \sum_{i \in I_{1}} G_{i i}^{(1)}(z) G_{i i}^{(1)}\left(z_{\mu}\right)+\frac{1}{b_{n}} \widetilde{\gamma_{n-1}}(z) \widetilde{\gamma_{n-1}}\left(z_{\mu}\right)\right] .
\end{aligned}
$$

Now using (36), we get

$$
\left.\left.\mid \mathbb{E}\left[\frac{1}{b_{n}} \widetilde{\gamma_{n-1}}(z) \widetilde{\gamma_{n-1}}\left(z_{\mu}\right)\right]\right)\right] \mid \leqslant \frac{1}{b_{n}} \sqrt{\mathbf{D} \sum_{i \in I_{1}} G_{i i}^{(1)}} \sqrt{\mathbf{D} \sum_{i \in I_{1}} G_{i i}^{(1)}}=O\left(\frac{1}{b_{n}}\right) .
$$

Letting $n \rightarrow \infty$, using (42) we have

$$
\begin{aligned}
\lim _{n \rightarrow \infty} D_{n}\left(z, z_{\mu}\right)=f^{2}(z) f^{2}\left(z_{\mu}\right)\left(1+2 f^{\prime}\left(z_{\mu}\right)\right) \\
\quad \times\left[\lim _{n \rightarrow \infty} \mathbb{E}\left[T_{n}\right]+\sigma^{2}+\kappa_{4} \lim _{n \rightarrow \infty} \frac{1}{b_{n}} \sum_{i \in I_{1}} \mathbb{E}\left[G_{i i}^{(1)}(z) G_{i i}^{(1)}\left(z_{\mu}\right)\right]\right] \\
\quad+f^{2}(z) f\left(z_{\mu}\right) \frac{d}{d z_{\mu}}\left[\lim _{n \rightarrow \infty} \mathbb{E}\left[T_{n}\right]+\kappa_{4} \lim _{n \rightarrow \infty} \frac{1}{b_{n}} \sum_{i \in I_{1}} \mathbb{E}\left[G_{i i}^{(1)}(z) G_{i i}^{(1)}\left(z_{\mu}\right)\right]\right],
\end{aligned}
$$

where

$$
T_{n}=\frac{2}{b_{n}} \sum_{i, j \in I_{1}} G_{i j}^{(1)}(z) G_{i j}^{(1)}\left(z_{\mu}\right) .
$$

Since $\mathbf{D}\left(G_{i i}\right)=O\left(1 / b_{n}\right)$ (see $\left.(36)\right)$, we have

$$
\begin{aligned}
\lim _{n \rightarrow \infty} \frac{1}{b_{n}} \sum_{i \in I_{1}} \mathbb{E}\left[G_{i i}^{(1)}(z) G_{i i}^{(1)}\left(z_{\mu}\right)\right] & =\lim _{n \rightarrow \infty} \frac{1}{b_{n}} \sum_{i \in I_{1}} \mathbb{E}\left[G_{i i}^{(1)}(z)\right] \mathbb{E}\left[G_{i i}^{(1)}\left(z_{\mu}\right)\right] \\
& =2 f(z) f\left(z_{\mu}\right) .
\end{aligned}
$$

We shall show in the subsection 4.1 of Appendix, that

$$
\lim _{n \rightarrow \infty} \mathbb{E}\left[T_{n}\right]=\frac{1}{4 \pi^{3}} \int_{-2 \sqrt{2}}^{2 \sqrt{2}} \int_{-2 \sqrt{2}}^{2 \sqrt{2}} \frac{\sqrt{8-x^{2}} \sqrt{8-y^{2}}}{(x-z)\left(y-z_{\mu}\right)} F(x, y) \mathbf{1}_{\{x \neq y\}} d x d y
$$

where

$$
F(x, y)=2 \int_{-\infty}^{\infty} \frac{u-u^{3}}{2\left(1-u^{2}\right)^{2}+u^{2}\left(x^{2}+y^{2}\right)-u\left(1+u^{2}\right) x y} d s
$$


where $u=(\sin s) / s$. Therefore,

$$
\begin{aligned}
& \lim _{n \rightarrow \infty} C_{n}\left(z_{\mu_{1}}, z_{\mu_{2}}\right)=\frac{1}{1-2 f^{2}\left(z_{\mu_{1}}\right)} \\
& \quad \times\left[f^{2}\left(z_{\mu_{1}}\right) f^{2}\left(z_{\mu_{2}}\right)\left(1+2 f^{\prime}\left(z_{\mu_{2}}\right)\right) \lim _{n \rightarrow \infty} \mathbb{E}\left[T_{n}\right]+f^{2}\left(z_{\mu_{1}}\right) f\left(z_{\mu_{2}}\right) \lim _{n \rightarrow \infty} \frac{d}{d z_{\mu_{2}}} \mathbb{E}\left[T_{n}\right]\right. \\
& \quad+\sigma^{2} f^{2}\left(z_{\mu_{1}}\right) f^{2}\left(z_{\mu_{2}}\right)\left(1+2 f^{\prime}\left(z_{\mu_{2}}\right)\right) \\
& \left.\quad+2 \kappa_{4}\left\{f^{3}\left(z_{\mu_{1}}\right) f^{3}\left(z_{\mu_{2}}\right)\left(1+2 f^{\prime}\left(z_{\mu_{2}}\right)\right)+f^{3}\left(z_{\mu_{1}}\right) f\left(z_{\mu_{2}}\right) f^{\prime}\left(z_{\mu_{2}}\right)\right\}\right] .
\end{aligned}
$$

Hence

$$
\begin{aligned}
V(\phi)= & \lim _{\eta \downarrow 0} \lim _{n \rightarrow \infty} V_{n}(\phi, \eta) \\
= & \frac{\kappa_{4}}{16 \pi^{2}}\left(\int_{-2 \sqrt{2}}^{2 \sqrt{2}} \frac{4-\mu^{2}}{\sqrt{8-\mu^{2}}} \phi(\mu) d \mu\right)^{2}+\frac{\sigma^{2}}{16 \pi^{2}}\left(\int_{-2 \sqrt{2}}^{2 \sqrt{2}} \frac{\mu \phi(\mu)}{\sqrt{8-\mu^{2}}} d \mu\right)^{2} \\
& +\int_{-2 \sqrt{2}}^{2 \sqrt{2}} \int_{-2 \sqrt{2}}^{2 \sqrt{2}} \sqrt{\left(8-x^{2}\right)\left(8-y^{2}\right)} F(x, y) \\
& \quad \times \int_{-2 \sqrt{2}}^{2 \sqrt{2}} \int_{-2 \sqrt{2}}^{2 \sqrt{2}} \frac{\mu_{1} \phi\left(\mu_{1}\right)}{\left(x-\mu_{1}\right) \sqrt{8-\mu_{1}^{2}}} \frac{\mu_{2} \phi\left(\mu_{2}\right)}{\left(x-\mu_{2}\right)^{2} \sqrt{8-\mu_{2}^{2}}} d \mu_{1} d \mu_{2} d x d y .
\end{aligned}
$$

This completes the proof of (10) and the proof of Theorem 1.

\section{Appendix.}

$\mathrm{Pr}$ o of of $\mathrm{Pr}$ op os it i o $\mathrm{n} 2$. Denote the averaging with respect to $\left\{w_{i j} ; 1 \leqslant i \leqslant k\right.$ or $\left.1 \leqslant j \leqslant n\right\}$ by $\mathbb{E}_{\leqslant k}$ and the averaging with respect to $\left\{w_{k j} ; 1 \leqslant j \leqslant n\right\}$ by $\mathbb{E}_{k}$. Using the martingale difference technique (see [6]), we have

$$
\begin{aligned}
\mathbf{D}\left\{\gamma_{n}\right\} & \leqslant \sum_{k=1}^{n} \mathbb{E}\left[\left|\mathbb{E}_{\leqslant k-1}\left[\gamma_{n}\right]-\mathbb{E}_{\leqslant k}\left[\gamma_{n}\right]\right|^{2}\right]=\sum_{k=1}^{n} \mathbb{E}\left[\left|\mathbb{E}_{\leqslant k-1}\left[\gamma_{n}-\mathbb{E}_{\leqslant k}\left[\gamma_{n}\right]\right]\right|^{2}\right] \\
& \leqslant \sum_{k=1}^{n} \mathbb{E}\left[\mathbb{E}_{\leqslant k-1}\left|\gamma_{n}-\mathbb{E}_{k}\left[\gamma_{n}\right]\right|^{2}\right]=\sum_{k=1}^{n} \mathbb{E}\left[\left|\gamma_{n}-\mathbb{E}_{k}\left[\gamma_{n}\right]\right|^{2}\right] .
\end{aligned}
$$

Note that

$$
\begin{aligned}
\mathbb{E}\left[\left|\gamma_{n}-\mathbb{E}_{1}\left[\gamma_{n}\right]\right|^{2}\right] & =\mathbb{E}\left[\left|\operatorname{Tr}(G)-\mathbb{E}_{1}[\operatorname{Tr}(G)]\right|^{2}\right] \\
& =\mathbb{E}\left[\left|\operatorname{Tr}(G)-\mathbb{E}_{1}[\operatorname{Tr}(G)]+\operatorname{Tr}\left(G^{(1)}\right)-\operatorname{Tr}\left(G^{(1)}\right)\right|^{2}\right] \\
& =\mathbb{E}\left[\left|\operatorname{Tr}\left(G-G^{(1)}\right)-\mathbb{E}_{1}\left[\operatorname{Tr}\left(G-G^{(1)}\right)\right]\right|^{2}\right] .
\end{aligned}
$$

From (32) we have

$$
\operatorname{Tr}\left(G-G^{(1)}\right)=-\frac{1+B(z)}{A(z)}
$$


where $A(z)=-G_{11}^{-1}, B(z)=\left\langle G^{(1)} G^{(1)} m^{(1)}, m^{(1)}\right\rangle$, and $G^{(1)}$ is defined in (16), and $m^{(1)}=\left(1 / \sqrt{b_{n}}\right)\left(w_{12}, w_{13}, \ldots, w_{1 n}\right)^{T}$. Indeed,

$$
\begin{aligned}
\mathbb{E}\left[\left|\gamma_{n}-\mathbb{E}_{1}\left[\gamma_{n}\right]\right|^{2}\right] & \leqslant \mathbb{E}\left[\left|\frac{1+B(z)}{A(z)}-\mathbb{E}_{1}\left[\frac{1+B(z)}{A(z)}\right]\right|^{2}\right] \\
& \leqslant 2 \mathbb{E}\left[\left|\frac{1}{A(z)}-\mathbb{E}_{1}\left[\frac{1}{A(z)}\right]\right|^{2}\right]+2 \mathbb{E}\left[\left|\frac{B(z)}{A(z)}-\mathbb{E}_{1}\left[\frac{B(z)}{A(z)}\right]\right|^{2}\right] .
\end{aligned}
$$

Now, by (21) and (25),

$$
\begin{aligned}
\mathbb{E}_{1}\left[\left|\frac{B(z)}{A(z)}-\mathbb{E}_{1}\left[\frac{B(z)}{A(z)}\right]\right|^{2}\right] & \leqslant \mathbb{E}_{1}\left[\left|\frac{B(z)}{A(z)}-\frac{\mathbb{E}_{1}[B(z)]}{E_{1}[A(z)]}\right|^{2}\right] \\
& \leqslant \mathbb{E}_{1}\left[\left|\frac{B_{1}^{\circ}}{\mathbb{E}_{1}[A]}-\frac{A_{1}^{\circ}}{\mathbb{E}_{1}[A]} \frac{B}{A}\right|^{2}\right] \\
& \leqslant 2 \mathbb{E}_{1}\left[\left|\frac{B_{1}^{\circ}}{\mathbb{E}_{1}[A]}\right|^{2}\right]+\frac{2}{|\operatorname{Im} z|^{2}} \mathbb{E}_{1}\left[\left|\frac{A_{1}^{\circ}}{\mathbb{E}_{1}[A]}\right|^{2}\right],
\end{aligned}
$$

where $A_{1}^{\circ}=A-\mathbb{E}_{1}[A]$. So it is enough to estimate $\mathbb{E}_{1}\left[\left|A_{1}^{\circ} / \mathbb{E}_{1}[A]\right|^{2}\right]$ and $\mathbb{E}_{1}\left[\left|B_{1}^{\circ} / \mathbb{E}_{1}[A]\right|^{2}\right]$. Note that

$$
\begin{aligned}
A & =z-\frac{1}{\sqrt{b_{n}}} w_{11}+\left\langle G^{(1)} m^{(1)}, m^{(1)}\right\rangle, \\
A_{1}^{\circ} & =-\frac{1}{\sqrt{b_{n}}} w_{11}+\frac{1}{b_{n}} \sum_{\substack{i \neq j \\
i, j \in I_{1}}} G_{i j}^{(1)} w_{1 i} w_{1 j}+\frac{1}{b_{n}} \sum_{i \in I_{1}} G_{i i}^{(1)}\left(w_{1 i}^{2}\right)^{\circ} .
\end{aligned}
$$

Therefore,

$$
\begin{aligned}
\mathbb{E}_{1}\left[\left|A_{1}^{\circ}\right|^{2}\right]= & \mathbb{E}_{1}\left[\frac{1}{b_{n}} w_{11}^{2}+\frac{1}{b_{n}^{2}} \sum_{\substack{i \neq j \\
i, j \in I_{1}}} G_{i j}^{(1)} w_{1 i} w_{1 j} \sum_{\substack{k \neq l \\
k, l \in I_{1}}} \overline{G_{k l}^{(1)}} w_{1 k} w_{1 l}\right. \\
& \left.+\frac{1}{b_{n}^{2}} \sum_{i \in I_{1}} G_{i i}^{(1)}\left(w_{1 i}^{2}\right)^{\circ} \sum_{l \in I_{1}} \overline{G_{l l}^{(1)}}\left(w_{1 l}^{2}\right)^{\circ}\right] \\
= & \frac{\sigma^{2}}{b_{n}}+\frac{2}{b_{n}^{2}} \sum_{\substack{i \neq j \\
i, j \in I_{1}}}\left|G_{i j}^{(1)}\right|^{2}+\frac{\mu_{4}-1}{b_{n}^{2}} \sum_{i \in I_{1}}\left|G_{i i}^{(1)}\right|^{2} \\
\leqslant & \frac{\sigma^{2}}{b_{n}}+\frac{2}{b_{n}^{2}} \frac{2 b_{n}}{|\operatorname{Im} z|^{2}}+\frac{\mu_{4}-1}{b_{n}^{2}} \frac{2 b_{n}}{|\operatorname{Im} z|^{2}} \\
\leqslant & \frac{1}{b_{n}}\left(\sigma^{2}+\frac{2+2 \mu_{4}}{|\operatorname{Im} z|^{2}}\right) .
\end{aligned}
$$

Now we want to estimate $\mathbb{E}_{1}\left[\left|B_{1}^{\circ}\right|^{2}\right]$, where $B=\left\langle G^{(1)} G^{(1)} m^{(1)}, m^{(1)}\right\rangle=$ $\left\langle H^{(1)} m^{(1)}, m^{(1)}\right\rangle$, and $B_{1}^{\circ}=B-\mathbb{E}_{1}[B]$. Therefore,

$$
\mathbb{E}_{1}[B]=\frac{1}{b_{n}} \sum_{i \in I_{1}} H_{i i}^{(1)}=\frac{1}{b_{n}} \sum_{i \in I_{1}} \sum_{j=2}^{n}\left(G_{i j}^{(1)}\right)^{2},
$$


and

$$
B_{1}^{\circ}=\frac{1}{b_{n}} \sum_{\substack{i \neq j \\ i, j \in I_{1}}} H_{i j}^{(1)} w_{1 i} w_{1 j}+\frac{1}{b_{n}} \sum_{i \in I_{1}} H_{i i}^{(1)}\left(w_{1 i}^{2}\right)^{\circ} .
$$

Let us call $C_{0}=\mathbb{E}\left[\left(w_{1 i}^{2}\right)^{\circ}\right]^{2}$. Then

$$
\begin{aligned}
\mathbb{E}_{1}\left[\left|B_{1}^{\circ}\right|^{2}\right] & =\frac{1}{b_{n}^{2}} \sum_{\substack{i \neq j \\
i, j \in I_{1}}}\left|H_{i j}^{(1)}\right|^{2}+\frac{C_{0}}{b_{n}^{2}} \sum_{i \in I_{1}}\left|H_{i i}^{(1)}\right|^{2} \\
& =\frac{1}{b_{n}^{2}} \sum_{\substack{i \neq j \\
i, j \in I_{1}}}\left|\sum_{k=2}^{n} G_{i k}^{(1)} G_{k j}^{(1)}\right|^{2}+\frac{C_{0}}{b_{n}^{2}} \sum_{i \in I_{1}}\left|\sum_{k=2}^{n} G_{i k}^{(1)} G_{k i}^{(1)}\right|^{2} \\
& \leqslant \frac{1}{b_{n}^{2}} \sum_{i \in I_{1}}\left\|\left(G^{(1)}\right)^{2}\right\|^{2}+\frac{C_{0}}{b_{n}^{2}} 2 b_{n}\left\|\left(G^{(1)}\right)^{2}\right\|^{2} \\
& =\frac{2}{b_{n}} \frac{1}{|\operatorname{Im} z|^{4}}+\frac{2 C_{0}}{b_{n}} \frac{1}{|\operatorname{Im} z|^{4}} \\
& =\frac{2\left(1+C_{0}\right)}{b_{n}|\operatorname{Im} z|^{4}} .
\end{aligned}
$$

We also have

$$
\mathbb{E}\left[\left|\frac{1}{A(z)}-\mathbb{E}_{1}\left[\frac{1}{A(z)}\right]\right|^{2}\right] \leqslant \frac{1}{|\operatorname{Im} z|^{2}} \mathbb{E}\left[\left|\frac{A_{1}^{\circ}}{\mathbb{E}_{1}[A]}\right|^{2}\right] .
$$

Note that $\mathbb{E}_{1}[A]=z+\left(1 / b_{n}\right) \sum_{i \in I_{1}} G_{i i}^{(1)}$. Since $\operatorname{Im} G_{i i}^{(1)}>0$, we have $\left|\mathbb{E}_{1}[A]\right| \geqslant$ $|\operatorname{Im} A| \geqslant y$. Also we know that $\left|G_{i i}^{(1)}\right| \leqslant 1 /|\operatorname{Im} z|=1 / y$. Therefore, $\left|\mathbb{E}_{1}[A]\right| \geqslant$ $|x|-2 / y$. Combining these we have

$$
\left|\mathbb{E}_{1}[A]\right|>\max \left\{y,|x|-\frac{2}{y}\right\} .
$$

Therefore,

$$
\begin{aligned}
\mathbb{E}\left[\left|\gamma_{n}-\mathbb{E}_{1}\left[\gamma_{n}\right]\right|^{2}\right] & \leqslant C_{1} \frac{2\left(1+C_{0}\right)}{b_{n}|\operatorname{Im} z|^{4}}\left|\mathbb{E}_{1}[A]\right|^{-2}+\frac{C_{2}}{b_{n}}\left(\sigma^{2}+\frac{2+2 \mu_{4}}{|\operatorname{Im} z|^{2}}\right) \frac{\left|\mathbb{E}_{1}[A]\right|^{-2}}{|\operatorname{Im} z|^{2}} \\
& \leqslant \frac{C}{b_{n}}\left(\frac{1}{|\operatorname{Im} z|^{2}}+\frac{1}{|\operatorname{Im} z|^{4}}\right)\left|\mathbb{E}_{1}[A]\right|^{-2}
\end{aligned}
$$

for some $C_{1}, C_{2}, C>0$ not depending on $z, n$. This implies

$$
\mathbf{D}\left(\gamma_{n}\right) \leqslant \frac{C n}{b_{n}}\left(\frac{1}{|\operatorname{Im} z|^{2}}+\frac{1}{|\operatorname{Im} z|^{4}}\right)\left(\max \left\{y,|x|-\frac{2}{y}\right\}\right)^{-2} \text {. }
$$

This completes the proof of Proposition 2.

Now we proceed to the proofs of the asymptotic estimates. All the asymptotic estimates listed in Lemma 1 and Lemma 2 hold uniformly in the set $\{z \in \mathbb{C}:|\operatorname{Im} z| \geqslant \eta\}$ for any given $\eta>0$. 
Lemma 1. Let $M$ be an $n \times n$ symmetric band matrix as defined in (2) which satisfies (3) and $\mathbb{E}\left[\left|w_{i j}\right|^{8}\right]$ is uniformly bounded. Then

(i)

$$
G_{i i}^{(1)}-G_{i i}=\frac{1}{A(z)}\left(G^{(1)} m^{(1)}\right)_{i}^{2}=\frac{1}{A(z)}\left(\frac{1}{\sqrt{b_{n}}} \sum_{j \in I_{1}} G_{i j}^{(1)} w_{1 j}\right)^{2},
$$

where $2 \leqslant i \leqslant n, A(z), m^{(1)}$, and $G^{(1)}$ are as defined in (14), (15), and (16).

(ii) $\left|\mathbb{E}\left[G_{i i}^{(1)}(z)\right]-\mathbb{E}\left[G_{i i}(z)\right]\right|=O\left(1 / b_{n}\right)$.

(iii)

$$
\begin{gathered}
\mathbb{E}\left[\left|G_{12}\right|^{2}\right]=O\left(\frac{1}{b_{n}}\right) \frac{1}{|\operatorname{Im} z|^{6}}, \quad \mathbb{E}\left[\left|G_{12}\right|^{4}\right]=O\left(\frac{1}{b_{n}^{2}}\right) \frac{1}{|\operatorname{Im} z|^{12}}, \\
\mathbb{E}\left[\left|G_{12}\right|^{8}\right]=O\left(\frac{1}{b_{n}^{4}}\right) \frac{1}{|\operatorname{Im} z|^{24}} .
\end{gathered}
$$

(iv) Let us denote the averaging with respect to $\left\{w_{1 i}\right\}_{1 \leqslant i \leqslant n}$ by $\mathbb{E}_{1}$. Then

$$
\begin{aligned}
b_{n} \mathbb{E}_{1}\left[A^{\circ}\left(z_{1}\right) A^{\circ}\left(z_{2}\right)\right]= & \sigma^{2}+\frac{2}{b_{n}} \sum_{i, j \in I_{1}} G_{i j}^{(1)}\left(z_{1}\right) G_{i j}^{(1)}\left(z_{2}\right) \\
& +\frac{\kappa_{4}}{b_{n}} \sum_{i \in I_{1}} G_{i i}^{(1)}\left(z_{1}\right) G_{i i}^{(1)}\left(z_{2}\right) \\
& +\frac{1}{b_{n}} \widetilde{\gamma_{n-1}}\left(z_{1}\right) \widetilde{\gamma_{n-1}}\left(z_{2}\right),
\end{aligned}
$$

where $\widetilde{\gamma_{n-1}}(z)=\sum_{i \in I_{1}}\left(G_{i i}^{(1)}-\mathbb{E}\left[G_{i i}^{(1)}(z)\right]\right)$ and $I_{1}=\left\{1<i \leqslant n:(1, i) \in I_{n}\right\}$.

(v)

$$
\begin{gathered}
\mathbb{E}_{1}\left[A^{\circ}\left(z_{1}\right) B^{\circ}\left(z_{2}\right)\right]=\frac{d}{d z_{2}} \mathbb{E}_{1}\left[A^{\circ}\left(z_{1}\right) A^{\circ}\left(z_{2}\right)\right], \text { where } \\
B\left(z_{2}\right)=\left\langle G^{(1)}\left(z_{2}\right) G^{(1)}\left(z_{2}\right) m^{(1)}, m^{(1)}\right\rangle .
\end{gathered}
$$

Lemma 2. Let $M$ be an $n \times n$ symmetric band matrix as defined in (2) which satisfies (3). Also assume that the probability distribution of $w_{j k}$ satisfies the Poincaré inequality with some uniform constant $m$ which does not depend on $n, j, k$. Then

(i)

$$
\mathbf{D}\left(\sum_{(1, i) \in I_{n}} G_{i i}\right)=O(1) \quad \text { and } \quad \mathbf{D}\left(G_{11}(z)\right)=O\left(\frac{1}{b_{n}}\right)
$$

(ii)

$$
\begin{aligned}
\mathbb{E}\left[\left|A^{\circ}\right|^{4}\right]= & O\left(\frac{1}{b_{n}^{2}}\right), \quad \mathbb{E}\left[\left|A^{\circ}\right|^{3}\right]=O\left(\frac{1}{b_{n}^{3 / 2}}\right), \\
& \mathbb{E}\left[\left|B^{\circ}\right|^{4}\right]=O\left(\frac{1}{b_{n}^{2}}\right) .
\end{aligned}
$$


(iii)

$$
\begin{gathered}
\mathbf{D}\left\{b_{n} \mathbb{E}_{1}\left[A^{\circ}\left(z_{1}\right) A^{\circ}\left(z_{2}\right)\right]\right\}=O\left(\frac{1}{b_{n}}\right) \text { and } \\
\mathbf{D}\left\{b_{n} \mathbb{E}_{1}\left[A^{\circ}\left(z_{1}\right) B^{\circ}\left(z_{2}\right)\right]\right\}=O\left(\frac{1}{b_{n}}\right) .
\end{gathered}
$$

(iv)

$$
\mathbb{E}\left[\left|\gamma_{n-1}^{\circ}(z)-\gamma_{n}^{\circ}(z)\right|^{4}\right]=O\left(\frac{1}{b_{n}^{2}}\right) \quad \text { and } \quad \mathbb{E}\left[\left|\gamma_{n}^{\circ}\right|^{4}\right]=O\left(\frac{n^{2}}{b_{n}^{2}}\right) .
$$

$\frac{1}{n} \mathbb{E}[\operatorname{Tr} G(z)]=f(z)+O\left(\frac{1}{|\operatorname{Im} z|^{6} b_{n}}\right), \quad$ where $f(z)=\frac{1}{4}\left(-z+\sqrt{z^{2}-8}\right)$.

$$
(\mathbb{E}[A(z)])^{-1}=-f(z)+O\left(b_{n}^{-1}\right) \quad \text { and } \quad \mathbb{E}[B(z)]=2 f^{\prime}(z)+O\left(b_{n}^{-1}\right) .
$$

Pro of of L e m ma 1. P r o of of (i). Suppose $\left(X_{1}, X_{2}, \ldots, X_{n}\right)$ is a $n$ dimensional normal random vector with a positive definite covariance matrix $A^{-1}$ and a mean $A^{-1} \underline{h}$, where $\underline{h} \in \mathbb{R}^{n}$. Then we have

$$
\begin{array}{r}
\int \exp \left[-\frac{1}{2}\langle A \underline{x}, \underline{x}\rangle+\langle\underline{h}, \underline{x}\rangle\right] d \underline{x}=(2 \pi)^{n / 2}|\operatorname{det} A|^{-1 / 2} \exp \left[\frac{1}{2}\left\langle A^{-1} \underline{h}, \underline{h}\right\rangle\right] \\
\frac{\int x_{i} x_{j} \exp \left[-\frac{1}{2}\langle A \underline{x}, \underline{x}\rangle+\langle\underline{h}, \underline{x}\rangle\right] d \underline{x}}{\int \exp \left[-\frac{1}{2}\langle A \underline{x}, \underline{x}\rangle+\langle\underline{h}, \underline{x}\rangle\right] d \underline{x}}=\left(A^{-1}\right)_{i j}+\left(A^{-1} \underline{h}\right)_{i}\left(A^{-1} \underline{h}\right)_{j}
\end{array}
$$

where $\underline{x}=\left(x_{1}, x_{2}, \ldots, x_{n}\right)^{T}$. In particular, for $\underline{h}=0$,

$$
\left(A^{-1}\right)_{i j}=\frac{\int x_{i} x_{j} \exp [-\langle A \underline{x}, \underline{x}\rangle / 2] d \underline{x}}{\int \exp [-\langle A \underline{x}, \underline{x}\rangle / 2] d \underline{x}} .
$$

Now doing the integrations in (45) with respect to all variables except $x_{1}$, and using (43) we get

$$
\begin{aligned}
\int \exp \left[-\frac{1}{2}\langle A \underline{x}, \underline{x}\rangle\right] d \underline{x}= & \int \exp \left[-\frac{a_{11} x_{1}^{2}}{2}\right] \\
& \times \int \exp \left[-\frac{1}{2}\left\langle A_{1} \underline{x}^{(1)}, \underline{x}^{(1)}\right\rangle-\left\langle x_{1} \underline{a}_{1}, \underline{x}^{(1)}\right\rangle\right] d \underline{x} \\
= & \frac{(2 \pi)^{(n-1) / 2}}{\left|\operatorname{det} A_{1}\right|^{1 / 2}} \int \exp \left[-\frac{x_{1}^{2}}{2}\left(a_{11}-\left\langle A_{1}^{-1} \underline{a_{1}}, \underline{a_{1}}\right\rangle\right)\right] d x_{1},
\end{aligned}
$$


where $\underline{x}^{(1)}=\left(x_{2}, x_{3}, \ldots, x_{n}\right)^{T}, \underline{a_{1}}=\left(a_{12}, a_{13}, \ldots, a_{1 n}\right)^{T}$ and $A_{1}=$ $\left(\left(A_{1}\right)_{i j}\right)_{i, j=2}^{n}$ is the $(n-1) \times(n-1)$ matrix obtained from $A$ after removing first row and first column, and for $i, j \neq 1$, using (44) and (43) we get

$$
\begin{aligned}
\int & x_{i} x_{j} \exp \left[-\frac{1}{2}\langle A \underline{x}, \underline{x}\rangle\right] d \underline{x} \\
= & \int \exp \left[-\frac{a_{11} x_{1}^{2}}{2}\right] \int x_{i} x_{j} \exp \left[-\frac{1}{2}\left\langle A_{1} \underline{x}^{(1)}, \underline{x}^{(1)}\right\rangle-\left\langle x_{1} \underline{a_{1}}, \underline{x}^{(1)}\right\rangle\right] d \underline{x}^{(1)} d x_{1} \\
= & \int \exp \left[-\frac{a_{11} x_{1}^{2}}{2}\right]\left[\left(A_{1}^{-1}\right)_{i j}+x_{1}^{2}\left(A_{1}^{-1} \underline{a_{1}}\right)_{i}\left(A_{1}^{-1} \underline{a}_{1}\right)_{j}\right] \\
& \times \int \exp \left[-\frac{1}{2}\left\langle A_{1} \underline{x}^{(1)}, \underline{x}^{(1)}\right\rangle-\left\langle x_{1} \underline{a}_{1}, \underline{x}^{(1)}\right\rangle\right] d \underline{x}^{(1)} d x_{1} \\
= & \frac{(2 \pi)^{(n-1) / 2}}{\left|\operatorname{det} A_{1}\right|^{1 / 2}} \int\left[\left(A_{1}^{-1}\right)_{i j}+x_{1}^{2}\left(A_{1}^{-1} \underline{a_{1}}\right)_{i}\left(A_{1}^{-1} \underline{a_{1}}\right)_{j}\right] \\
& \times \exp \left[-\frac{x_{1}^{2}}{2}\left(a_{11}-\left\langle A_{1}^{-1} \underline{a}_{1}, \underline{a}_{1}\right\rangle\right)\right] d x_{1} .
\end{aligned}
$$

Therefore, from (45) we get

$$
\begin{aligned}
\left(A^{-1}\right)_{i j}= & \left(A_{1}^{-1}\right)_{i j}+\left(A_{1}^{-1} \underline{a_{1}}\right)_{i}\left(A_{1}^{-1} \underline{a_{1}}\right)_{j} \\
& \times \frac{\int x_{1}^{2} \exp \left[-x_{1}^{2}\left(a_{11}-\left\langle A_{1}^{-1} \underline{a_{1}}, \underline{a_{1}}\right\rangle\right) / 2\right] d x_{1}}{\int \exp \left[-x_{1}^{2}\left(a_{11}-\left\langle A_{1}^{-1} \underline{a_{1}}, \underline{a_{1}}\right\rangle\right) / 2\right] d x_{1}} \\
= & \left(A_{1}^{-1}\right)_{i j}+\frac{\left(A_{1}^{-1} \underline{a_{1}}\right)_{i}\left(A_{1}^{-1} \underline{a_{1}}\right)_{j}}{a_{11}-\left\langle A_{1}^{-1} \underline{a_{1}}, \underline{a_{1}}\right\rangle} .
\end{aligned}
$$

Applying the above formula for $A=(M-z I)$, where $z \in \mathbb{R},|z|>\|M\|$, we obtain

$$
G_{i j}=G_{i j}^{(1)}+\frac{\left(G^{(1)} m^{(1)}\right)_{i}\left(G^{(1)} m^{(1)}\right)_{j}}{w_{11} / \sqrt{b_{n}}-z-\left\langle G^{(1)} m^{(1)}, m^{(1)}\right\rangle}, \quad i, j \geqslant 2,
$$

where $m^{(1)}, G^{(1)}$ are as defined in (15), (16), respectively. From the above formula we obtain

$$
G_{i i}-G_{i i}^{(1)}=-\frac{\left(G^{(1)} m^{(1)}\right)_{i}^{2}}{A(z)}, \quad \text { for all } 2 \leqslant i \leqslant n,
$$

where $A(z)$ is as defined in (14). The above is true for all $z \in \mathbb{R}$ such that $|z|>\|M\|$. By analytic continuity one can extend it to the whole complex plane. This completes the proof.

P r o of of (i i). Recall $I_{1}=\left\{1<i \leqslant n:(1, i) \in I_{n}\right\}$. Now using (32) and (21) we have

$$
\left|\mathbb{E}\left[G_{i i}^{(1)}(z)\right]-\mathbb{E}\left[G_{i i}(z)\right]\right|=\left|\mathbb{E}\left[\frac{1}{A}\left(G^{(1)} m^{(1)}\right)_{i}^{2}\right]\right|
$$




$$
\begin{aligned}
& =\left|\mathbb{E}\left[\frac{1}{A}\left(\frac{1}{\sqrt{b_{n}}} \sum_{j \in I_{1}} G_{i j}^{(1)} w_{1 j}\right)^{2}\right]\right| \\
& \leqslant \frac{1}{b_{n}} \frac{1}{|\operatorname{Im} z|} \mathbb{E}\left[\left|\sum_{j \in I_{1}} G_{i j}^{(1)} w_{1 j}\right|^{2}\right] \\
& \leqslant \frac{1}{b_{n}|\operatorname{Im} z|} \mathbb{E}\left[\sum_{j \in I_{1}}\left|G_{i j}^{(1)}\right|^{2} w_{1 j}^{2}+\sum_{j_{1} \neq j_{2} \in I_{1}} G_{i j_{1}}^{(1)} \overline{G_{i j_{2}}^{(1)}} w_{1 j_{1}} w_{1 j_{2}}\right] \\
& =\frac{1}{b_{n}|\operatorname{Im} z|} \mathbb{E} \mathbb{E}_{1}\left[\sum_{j \in I_{1}}\left|G_{i j}^{(1)}\right|^{2} w_{1 j}^{2}+\sum_{j_{1} \neq j_{2} \in I_{1}} G_{i j_{1}}^{(1)} \overline{G_{i j_{2}}^{(1)}} w_{1 j_{1}} w_{1 j_{2}}\right] \\
& =\frac{1}{b_{n}|\operatorname{Im} z|} \mathbb{E}\left[\sum_{j \in I_{1}}\left|G_{1 j}^{(1)}\right|^{2}\right] \\
& \leqslant \frac{1}{b_{n}|\operatorname{Im} z|} \mathbb{E}\left\|G^{(1)}\right\|^{2} \leqslant \frac{1}{b_{n}|\operatorname{Im} z|^{3}} .
\end{aligned}
$$

P r o o f o f (i i i). Using the resolvent formula given in [7], we have

$$
G_{12}=-G_{22} G_{11}^{(2)} K_{12}^{(12)}
$$

where $G^{(2)}$ is the resolvent of the $(n-1) \times(n-1)$ minor obtained by removing the $k$ th row and $k$ th column from the matrix $M$,

$$
\begin{gathered}
K_{12}^{(12)}=m_{12}-m_{(1)} G^{(12)} m_{(2)} \\
m_{(1)}=\frac{1}{\sqrt{b_{n}}}\left(w_{13}, w_{14}, \ldots, w_{1 n}\right), \quad m_{(2)}=\frac{1}{\sqrt{b_{n}}}\left(w_{23}, w_{24}, \ldots, w_{2 n}\right)^{T}
\end{gathered}
$$

$G^{(i j)}=\left(M^{(i j)}-z I\right)^{-1}$, and $M^{(i j)}$ is $(n-2) \times(n-2)$ matrix obtained from $M$ after removing $i$ th and $j$ th rows and columns. Therefore,

$$
\begin{aligned}
\mathbb{E}\left[\left|G_{12}\right|^{2}\right] & =\mathbb{E}\left[\left|G_{22} G_{11}^{(2)} K_{12}^{(12)}\right|^{2}\right] \\
& \leqslant \frac{1}{|\operatorname{Im} z|^{2}} \frac{1}{|\operatorname{Im} z|^{2}} \mathbb{E}\left[\left|m_{12}-m_{(1)} G^{(12)} m_{(2)}\right|^{2}\right] \\
& =\frac{1}{|\operatorname{Im} z|^{4}} \mathbb{E}\left[\left|\frac{w_{12}}{\sqrt{b_{n}}}-\frac{1}{b_{n}} \sum_{\substack{(1, i),(2, j) \in I_{n} \\
i, j \neq 1,2}} G_{i j}^{(12)} w_{1 i} w_{2 j}\right|^{2}\right] \\
& \leqslant \frac{1}{|\operatorname{Im} z|^{4}} \mathbb{E} \mathbb{E}_{\leqslant 2}\left[\frac{w_{12}^{2}}{b_{n}}+\frac{1}{b_{n}^{2}} \sum_{\substack{(1, i),(2, j) \in I_{n} \\
i, j \neq 1,2}}\left|G_{i j}^{(12)}\right|^{2} w_{1 i}^{2} w_{2 j}^{2}\right] \\
& \leqslant \frac{1}{|\operatorname{Im} z|^{4}} \mathbb{E}\left[\frac{1}{b_{n}}+\frac{1}{b_{n}^{2}} \sum_{i, j}\left|G_{i j}^{(12)}\right|^{2} \mathbb{E}_{\leqslant 2}\left[w_{1 i}^{2}\right] \mathbb{E}_{\leqslant 2}\left[w_{2 j}^{2}\right]\right] \\
& \leqslant \frac{1}{|\operatorname{Im} z|^{4}} \mathbb{E}\left[\frac{1}{b_{n}}+\frac{1}{b_{n}^{2}} \frac{b_{n}}{|\operatorname{Im} z|^{2}}\right]=O\left(\frac{1}{b_{n}}\right) \frac{1}{|\operatorname{Im} z|^{6}},
\end{aligned}
$$


where $E_{\leqslant 2}$ is the averaging with respect to the first two rows and columns. Similarly, we can prove that

$\mathbb{E}\left[\left|G_{12}\right|^{4}\right]=O\left(\frac{1}{b_{n}^{2}}\right) \frac{1}{|\operatorname{Im} z|^{12}}, \quad$ and $\quad \mathbb{E}\left[\left|G_{12}\right|^{8}\right]=O\left(\frac{1}{b_{n}^{4}}\right) \frac{1}{|\operatorname{Im} z|^{24}}$.

P r o of of (iv). We know that

$$
\begin{aligned}
A\left(z_{1}\right) & =z_{1}-\frac{w_{11}}{\sqrt{b_{n}}}+\left\langle G^{(1)} m^{(1)}, m^{(1)}\right\rangle, \\
A^{\circ}\left(z_{1}\right) & =-\frac{w_{11}}{\sqrt{b_{n}}}+\frac{1}{b_{n}} \sum_{i \neq j \in I_{1}} G_{i j}^{(1)} w_{1 i} w_{1 j}+\frac{1}{b_{n}} \sum_{i \in I_{1}} G_{i i}^{(1)} w_{1 i}^{2}-\frac{1}{b_{n}} \sum_{i \in I_{1}} \mathbb{E}\left[G_{i i}^{(1)}\right] .
\end{aligned}
$$

Now we can estimate

$$
\begin{aligned}
& b_{n} \mathbb{E}_{1}\left[A^{\circ}\left(z_{1}\right) A^{\circ}\left(z_{2}\right)\right] \\
& =\sigma^{2}+\frac{1}{b_{n}} \mathbb{E}_{1}\left[\sum_{\substack{i_{1} \neq j_{1} \in I_{1} \\
i_{2} \neq j_{2} \in I_{1}}} G_{i_{1} j_{1}}^{(1)}\left(z_{1}\right) w_{1 i_{1}} w_{1 j_{1}} G_{i_{2} j_{2}}^{(1)}\left(z_{2}\right) w_{1 i_{2}} w_{1 j_{2}}\right] \\
& +\frac{1}{b_{n}} \mathbb{E}_{1}\left[\sum_{i, j \in I_{1}} G_{i i}^{(1)}\left(z_{1}\right) G_{j j}^{(1)}\left(z_{2}\right) w_{1 i}^{2} w_{1 j}^{2}\right] \\
& -\frac{1}{b_{n}} \mathbb{E}\left[\sum_{i \in I_{1}} G_{i i}^{(1)}\left(z_{2}\right)\right] \mathbb{E}_{1}\left[\sum_{i \in I_{1}} G_{i i}^{(1)}\left(z_{1}\right) w_{1 i}^{2}\right] \\
& -\frac{1}{b_{n}} \mathbb{E}\left[\sum_{i \in I_{1}} G_{i i}^{(1)}\left(z_{1}\right)\right] \mathbb{E}_{1}\left[\sum_{i \in I_{1}} G_{i i}^{(1)}\left(z_{2}\right) w_{1 i}^{2}\right] \\
& +\frac{1}{b_{n}} \mathbb{E}\left[\sum_{i \in I_{1}} G_{i i}^{(1)}\left(z_{1}\right)\right] \mathbb{E}\left[\sum_{i \in I_{1}} G_{i i}^{(1)}\left(z_{2}\right)\right] \\
& =\sigma^{2}+\frac{2}{b_{n}} \sum_{i \neq j \in I_{1}} G_{i j}^{(1)}\left(z_{1}\right) G_{i j}^{(1)}\left(z_{2}\right)+\frac{1}{b_{n}} \sum_{i \neq j \in I_{1}} G_{i i}^{(1)}\left(z_{1}\right) G_{j j}^{(1)}\left(z_{2}\right) \\
& +\frac{\mu_{4}}{b_{n}} \sum_{i \in I_{1}} G_{i i}^{(1)}\left(z_{1}\right) G_{i i}^{(1)}\left(z_{2}\right)+\frac{1}{b_{n}} \widetilde{\gamma_{n-1}}\left(z_{1}\right) \widetilde{\gamma_{n-1}}\left(z_{2}\right) \\
& -\frac{1}{b_{n}}\left(\sum_{i \in I_{1}} G_{i i}^{(1)}\left(z_{1}\right)\right)\left(\sum_{i \in I_{1}} G_{i i}^{(1)}\left(z_{2}\right)\right) \\
& =\sigma^{2}+\frac{2}{b_{n}} \sum_{i, j \in I_{1}} G_{i j}^{(1)}\left(z_{1}\right) G_{i j}^{(1)}\left(z_{2}\right)+\frac{\mu_{4}}{b_{n}} \sum_{i \in I_{1}} G_{i i}^{(1)}\left(z_{1}\right) G_{i i}^{(1)}\left(z_{2}\right) \\
& -\frac{3}{b_{n}} \sum_{i \in I_{1}} G_{i i}^{(1)}\left(z_{1}\right) G_{i i}^{(1)}\left(z_{2}\right)+\frac{1}{b_{n}} \widetilde{\gamma_{n-1}}\left(z_{1}\right) \widetilde{\gamma_{n-1}}\left(z_{2}\right) \\
& =\sigma^{2}+\frac{2}{b_{n}} \sum_{i, j \in I_{1}} G_{i j}^{(1)}\left(z_{1}\right) G_{i j}^{(1)}\left(z_{2}\right)+\frac{\kappa_{4}}{b_{n}} \sum_{i \in I_{1}} G_{i i}^{(1)}\left(z_{1}\right) G_{i i}^{(1)}\left(z_{2}\right) \\
& +\frac{1}{b_{n}} \widetilde{\gamma_{n-1}}\left(z_{1}\right) \widetilde{\gamma_{n-1}}\left(z_{2}\right)
\end{aligned}
$$


where $\kappa_{4}=\mu_{4}-3$.

P r o of of $(v)$. Observe that

$$
\begin{aligned}
B\left(z_{2}\right) & =\left\langle G^{(1)} G^{(1)} m^{(1)}, m^{(1)}\right\rangle=\frac{1}{b_{n}} \sum_{i, j \in I_{1}}\left(G^{(1)} G^{(1)}\right)_{i j} w_{1 i} w_{1 j} \\
& =\frac{1}{b_{n}} \sum_{i, j \in I_{1}} \sum_{k=2}^{n} G_{i k}^{(1)} G_{k j}^{(1)} w_{1 i} w_{1 j},
\end{aligned}
$$

and

$$
\frac{d}{d z_{2}} G_{i j}^{(1)}\left(z_{2}\right)=\left(G^{(1)}\left(z_{2}\right) G^{(1)}\left(z_{2}\right)\right)_{i j}=\sum_{k=2}^{n} G_{i k}^{(1)}\left(z_{2}\right) G_{k j}^{(1)}\left(z_{2}\right) .
$$

Now, proceed as in (iv) and use the above facts to prove the result. Here we skip the details.

Pro of of $\mathrm{E}$ e m m a 2. Pro of of (i). Since $w_{j k}$ satisfies the Poincaré inequality with constant $m$ and the Poincaré inequality tensorises, the joint distribution of $\left\{w_{j k}\right\}_{(j, k) \in I_{n}^{+}}$on $\mathbb{R}^{n\left(b_{n}+1\right)}$ satisfies the Poincaré inequality with same constant $m$. Therefore, we have

$$
\mathbf{D}\left(\Phi\left(\left\{w_{j k}\right\}_{(j, k) \in I_{n}^{+}}\right)\right) \leqslant \frac{1}{m} \sum_{(j, k) \in I_{n}^{+}} \mathbb{E}\left[\left|\frac{\partial \Phi}{\partial w_{j k}}\right|^{2}\right],
$$

for any continuously differentiable function $\Phi$. Therefore,

$$
\begin{aligned}
\mathbf{D}\left(\sum_{(1, i) \in I_{n}} G_{i i}\right) & \leqslant \frac{1}{m} \sum_{(j, k) \in I_{n}^{+}} \mathbb{E}\left[\left|\frac{\partial}{\partial w_{j k}} \sum_{(1, i) \in I_{n}} G_{i i}\right|^{2}\right] \\
& \leqslant \frac{4}{m b_{n}} \sum_{(j, k) \in I_{n}^{+}} \mathbb{E}\left[\left|\sum_{(1, i) \in I_{n}} G_{i j} G_{k i}\right|^{2}\right] \\
& =\frac{4}{m b_{n}} \sum_{(j, k) \in I_{n}^{+}} \mathbb{E}\left[\left|\alpha_{k j}\right|^{2}\right] \quad \text { where } \alpha_{k j}=\sum_{(1, i) \in I_{n}} G_{k i} G_{i j} \\
& \leqslant \frac{4}{m b_{n}} \sum_{j, k=1}^{n} \mathbb{E}\left[\left|\alpha_{k j}\right|^{2}\right] \\
& =\frac{4}{m b_{n}} \mathbb{E}\left[\left\|V V^{T}\right\|_{F b}^{2}\right] \\
& =\frac{4}{m b_{n}} \mathbb{E}\left[\sum_{i=1}^{n}\left|\beta_{i}\right|^{2}\right]
\end{aligned}
$$

where

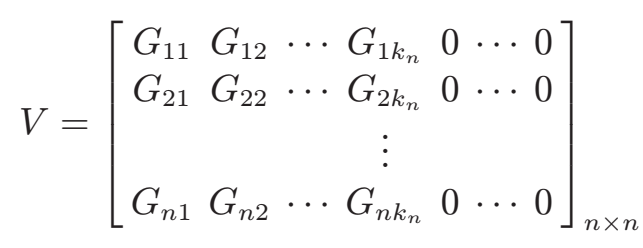


and $\|\cdot\|_{F b}$ stands for the Frobenius norm, and $\beta_{i}$ s are the eigenvalues of $V V^{T}$. Here, we denote the set $\left\{i:(1, i) \in I_{n}\right\}$ by $\left\{1,2, \ldots, k_{n}\right\}$. Observe that $k_{n}=2 b_{n}+1$. Since $\operatorname{rank}\left(V V^{T}\right) \leqslant k_{n}=O\left(b_{n}\right)$, we have $\#\left\{i: \beta_{i} \neq 0\right\} \leqslant$ $k_{n}=O\left(b_{n}\right)$. Also we know that $\|V\| \leqslant\|G\|$. Therefore,

$$
\left|\beta_{i}\right|^{2} \leqslant\left\|V V^{T}\right\|^{2} \leqslant\|G\|^{4} \leqslant \frac{1}{|\operatorname{Im} z|^{4}} .
$$

Consequently, we have

$$
\mathbf{D}\left(\sum_{(1, i) \in I_{n}} G_{i i}\right) \leqslant \frac{4}{m b_{n}} \mathbb{E}\left[\sum_{i=1}^{n}\left|\beta_{i}\right|^{2}\right] \leqslant \frac{4}{m b_{n}} \frac{O\left(b_{n}\right)}{|\operatorname{Im} z|^{4}}=O(1) .
$$

This completes proof of first part of (36).

Recall the definition of $A$ from (14), $A=z-b_{n}^{-1 / 2} w_{11}+\left(G^{(1)} m^{(1)}, m^{(1)}\right)$. Then

$$
\begin{aligned}
A^{\circ} & =A-\mathbb{E}[A] \\
& =-\frac{1}{\sqrt{b_{n}}} w_{11}+\frac{1}{b_{n}} \sum_{\substack{i \neq j \\
i, j \in I_{1}}} G_{i j}^{(1)} w_{1 i} w_{1 j}+\frac{1}{b_{n}} \sum_{i \in I_{1}}\left(G_{i i}^{(1)} w_{1 i}^{2}-\mathbb{E}\left[G_{i i}^{(1)}\right]\right),
\end{aligned}
$$

Consider

$$
\begin{aligned}
A_{1}^{\circ} & =A-\mathbb{E}_{1}[A] \\
& =-\frac{1}{\sqrt{b_{n}}} w_{11}+\frac{1}{b_{n}} \sum_{\substack{i \neq j \\
i, j \in I_{1}}} G_{i j}^{(1)} w_{1 i} w_{1 j}+\frac{1}{b_{n}} \sum_{i \in I_{1}}\left(G_{i i}^{(1)} w_{1 i}^{2}-G_{i i}^{(1)}\right) .
\end{aligned}
$$

So we have

$$
A^{\circ}-A_{1}^{\circ}=\frac{1}{b_{n}} \sum_{i \in I_{1}}\left(G_{i i}^{(1)}-\mathbb{E}\left[G_{i i}^{(1)}\right]\right)=: \frac{1}{b_{n}} \widetilde{\gamma_{n-1}} .
$$

Hence

$$
\mathbb{E}\left[\left|A^{\circ}\right|^{2}\right]=\mathbb{E}\left[\left|A_{1}^{\circ}+b_{n}^{-1} \widetilde{\gamma_{n-1}}\right|^{2}\right] \leqslant 2\left[\mathbb{E}\left[\left|A_{1}^{\circ}\right|^{2}\right]+\frac{1}{b_{n}^{2}} \mathbb{E}\left[\left|\widetilde{\gamma_{n-1}}\right|^{2}\right]\right] .
$$

From (31), we know that $\mathbb{E}\left[\left|A_{1}^{\circ}\right|^{2}\right]=O\left(1 / b_{n}\right)$ and from (47), We have $\mathbb{E}\left[\left|\widetilde{\gamma_{n-1}}\right|^{2}\right]=O(1)$. Combining these two facts and using (17), we have

$$
\mathbf{D}\left(G_{11}(z)\right)=\mathbb{E}\left|\frac{1}{A}-\mathbb{E} \frac{1}{A}\right|^{2} \leqslant \mathbb{E}\left|\frac{1}{A}-\frac{1}{\mathbb{E} A}\right|^{2}=\mathbb{E}\left|\frac{A^{\circ}}{A \mathbb{E} A}\right|^{2}=O\left(\frac{1}{b_{n}}\right) .
$$

This completes the proof of second part.

P r o o f o f (i i). Proof of (37). Recall from (48)

$$
A_{1}^{\circ}=-\frac{w_{11}}{\sqrt{b_{n}}}+\frac{1}{b_{n}} \sum_{i \neq j \in I_{1}} G_{i j}^{(1)} w_{1 i} w_{1 j}+\frac{1}{b_{n}} \sum_{i \in I_{1}} G_{i i}^{(1)}\left(w_{1 i}^{2}\right)^{\circ}=: T_{1}+T_{2}+T_{3} .
$$


We have $\mathbb{E}\left[\left|T_{1}\right|^{4}\right]=O\left(1 / b_{n}^{2}\right)$. Now

$\mathbb{E}\left[\left|T_{2}\right|^{4}\right]=\frac{1}{b_{n}^{4}} \mathbb{E}\left[\sum_{i \neq j, k \neq l, p \neq q, s \neq t \in I_{1}} G_{i j}^{(1)} \overline{G_{k l}^{(1)}} G_{p q}^{(1)} \overline{G_{s t}^{(1)}} w_{1 i} w_{1 j} w_{1 k} w_{1 l} w_{1 p} w_{1 q} w_{1 s} w_{1 t}\right]$.

We use the similar technique as the moment method in the proof of the semicircle law. In the above sum of expectations, we have nonzero terms if the indices of $w_{1 m}$ 's match in a certain way. Nonzero contribution to $\mathbb{E}\left[\left|T_{2}\right|^{4}\right]$ come from the two types of matches.

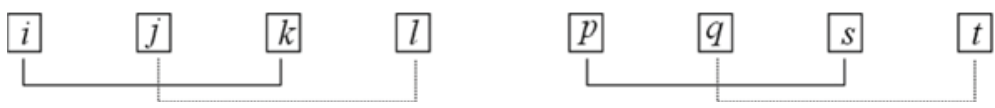

Fig. 1. Type I matching.

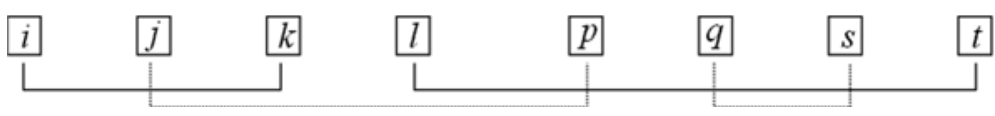

Fig. 2. Type II matching.

Type I. Contribution from this kind of matching is

$$
\begin{aligned}
\frac{1}{b_{n}^{4}} \mathbb{E} & {\left[\sum_{i \neq j, p \neq k \in I_{1}}\left|G_{i j}^{(1)}\right|^{2}\left|G_{p q}^{(1)}\right|^{2} w_{1 i}^{2} w_{1 j}^{2} w_{1 p}^{2} w_{1 q}^{2}\right] } \\
& =\frac{1}{b_{n}^{4}} \mathbb{E}_{1}\left[\sum_{i \neq j, p \neq k \in I_{1}}\left|G_{i j}^{(1)}\right|^{2}\left|G_{p q}^{(1)}\right|^{2} w_{1 i}^{2} w_{1 j}^{2} w_{1 p}^{2} w_{1 q}^{2}\right] \\
& =\frac{1}{b_{n}^{4}} \sum_{i \neq j} \sum_{p \neq q} \mathbb{E}\left[\left|G_{i j}^{(1)}\right|^{2}\left|G_{p q}^{(1)}\right|^{2}\right] \\
& \leqslant \frac{1}{b_{n}^{4}} \sum_{i \neq j} \sum_{p \neq q} \sqrt{\mathbb{E}\left[\left|G_{i j}^{(1)}\right| 4\right] \mathbb{E}\left[\left|G_{p q}^{(1)}\right|^{4}\right]} \\
& =\frac{1}{b_{n}^{4}} \sum_{i \neq j} \sum_{p \neq q} O\left(\frac{1}{b_{n}^{2}}\right) \quad(\mathrm{using}(33)) \\
& =O\left(\frac{1}{b_{n}^{2}}\right) .
\end{aligned}
$$

Type II. Similarly, contribution from the type II matching is

$$
\frac{1}{b_{n}^{4}} \mathbb{E}\left[\sum_{i \neq j} \sum_{q \neq l \in I_{1}} G_{i j}^{(1)} \overline{G_{i l}^{(1)}} G_{j q}^{(1)} \overline{G_{q l}^{(1)}} w_{1 i}^{2} w_{1 j}^{2} w_{1 q}^{2} w_{1 l}^{2}\right]=O\left(\frac{1}{b_{n}^{2}}\right) .
$$

Similarly, $\mathbb{E}\left[\left|T_{3}\right|^{4}\right]=O\left(1 / b_{n}^{2}\right)$. Hence

$$
\mathbb{E}\left[\left|A_{1}^{\circ}\right|^{4}\right]=O\left(\frac{1}{b_{n}^{2}}\right) .
$$


Using Lemma 4.4.3. from [1] with the help of the Poincaré inequality, we have $\mathbb{E}\left[\left|\widetilde{\gamma_{n-1}}\right|^{4}\right] \leqslant C\|\| \nabla \widetilde{\gamma_{n-1}}\left\|_{2}\right\|_{\infty}^{4}$, where $C$ is a constant depends only on the constant $m$ of the Poincaré inequality. Following the arguments given at the right side of (46) onward and (47), one can show that $\left\|\nabla \widetilde{\gamma_{n-1}}\right\|_{2} \leqslant C /|\operatorname{Im} z|^{4}$, where $C$ depends only on $m$. Hence $\mathbb{E}\left[\left|\widetilde{\gamma_{n-1}}\right|^{4}\right]=O(1)$. Consequently, using relation (49) and (51), we have $\mathbb{E}\left[\left|A^{\circ}\right|^{4}\right]=O\left(1 / b_{n}^{2}\right)$. Then $\mathbb{E}\left[\left|A^{\circ}\right|^{3}\right] \leqslant$ $\left(\mathbb{E}\left[\left|A^{\circ}\right|^{4}\right]\right)^{3 / 4}=O\left(1 / b_{n}^{3 / 2}\right)$.

P r o o f of (38). First we write $B$ as

$$
B=\left\langle G^{(1)} G^{(1)} m^{(1)}, m^{(1)}\right\rangle=\left\langle H^{(1)} m^{(1)}, m^{(1)}\right\rangle=\frac{1}{b_{n}} \sum_{i, j \in I_{1}} H_{i j}^{(1)} w_{1 i} w_{1 j},
$$

where $H^{(1)}=G^{(1)} G^{(1)}$. Define

$$
B_{1}^{\circ}:=\frac{1}{b_{n}} \sum_{i \neq j \in I_{1}} H_{i j}^{(1)} w_{1 i} w_{1 j}+\frac{1}{b_{n}} \sum_{i \in I_{1}} H_{i i}^{(1)}\left(w_{1 i}^{2}\right)^{\circ} .
$$

Then we can write

$$
\begin{aligned}
B^{\circ} & =B-\mathbb{E}[B] \\
& =\frac{1}{b_{n}} \sum_{i \neq j \in I_{1}} H_{i j} w_{1 i} w_{1 j}+\frac{1}{b_{n}} \sum_{i \in I_{1}}\left[H_{i i}^{(1)} w_{1 i}^{2}-\mathbb{E}\left[H_{i i}^{(1)}\right]\right] \\
& =B_{1}^{\circ}+\frac{1}{b_{n}} \sum_{i \in I_{1}}\left(H_{i i}^{(1)}-\mathbb{E},\left[H_{i i}^{(1)}\right]\right) \\
& =B_{1}^{\circ}+\frac{1}{b_{n}} \overline{\gamma_{n-1}}
\end{aligned}
$$

where

$$
\begin{aligned}
\overline{\gamma_{n-1}}(z)=\sum_{i \in I_{1}}\left(H_{i i}^{(1)}-\mathbb{E}\left[H_{i i}^{(1)}\right]\right) & =\sum_{i \in I_{1}} \sum_{j=2}^{n}\left(G_{i j}^{(1)} G_{j i}^{(1)}-\mathbb{E}\left[G_{i j}^{(1)} G_{j i}^{(1)}\right]\right) \\
& =\frac{d}{d z} \widetilde{\gamma_{n-1}}(z) .
\end{aligned}
$$

Proceeding as in the estimate of $\mathbb{E}\left[\left|A_{1}^{\circ}\right|^{4}\right]$, we can show

$$
\mathbb{E}\left[\left|B_{1}^{\circ}\right|^{4}\right]=O\left(\frac{1}{b_{n}^{2}}\right) .
$$

We have shown that $\mathbb{E}\left[\left|\widetilde{\gamma_{n-1}}(z)\right|^{4}\right]=O(1)$. Using this fact and Cauchy's theorem we have $\mathbb{E}\left[\left|\overline{\gamma_{n-1}}(z)\right|^{4}\right]=O(1)$. Hence we have the result.

Proof of (i i i).

$$
\begin{aligned}
\mathbf{D}\left\{b_{n} \mathbb{E}_{1}\left[A^{\circ}\left(z_{1}\right) A^{\circ}\left(z_{2}\right)\right]\right\}= & \mathbf{D}\left(T_{1}\right)+\mathbf{D}\left(T_{2}\right)+\mathbf{D}\left(T_{3}\right) \\
& +2 \operatorname{Cov}\left(T_{1}, T_{2}\right)+2 \operatorname{Cov}\left(T_{2}, T_{3}\right)+2 \operatorname{Cov}\left(T_{3}, T_{1}\right),
\end{aligned}
$$


where

$$
\begin{gathered}
T_{1}=\frac{2}{b_{n}} \sum_{i, j \in I_{1}} G_{i j}^{(1)}\left(z_{1}\right) G_{i j}^{(1)}\left(z_{2}\right), \quad T_{2}=\frac{\kappa_{4}}{b_{n}} \sum_{i \in I_{1}} G_{i i}^{(1)}\left(z_{1}\right) G_{i i}^{(1)}\left(z_{2}\right), \\
T_{3}=\frac{1}{b_{n}} \widetilde{\gamma_{n-1}}\left(z_{1}\right) \widetilde{\gamma_{n-1}}\left(z_{2}\right) .
\end{gathered}
$$

Now,

$$
\mathbf{D}\left(T_{2}\right)=\frac{\kappa_{4}^{2}}{b_{n}^{2}} \mathbf{D}\left\{\sum_{i \in I_{1}} G_{i i}^{(1)}\left(z_{1}\right) G_{i i}^{(1)}\left(z_{2}\right)\right\}
$$

and

$$
\begin{aligned}
\mathbf{D}\left\{G_{i i}^{(1)}\left(z_{1}\right) G_{i i}^{(1)}\left(z_{2}\right)\right\} & =\mathbb{E}\left|G_{i i}^{(1)}\left(z_{1}\right) G_{i i}^{(1)}\left(z_{2}\right)-\mathbb{E}\left[G_{i i}^{(1)}\left(z_{1}\right) G_{i i}^{(1)}\left(z_{2}\right)\right]\right|^{2} \\
& \leqslant \frac{2}{\left|\operatorname{Im} z_{1}\right|^{2}} \mathbf{D}\left(G_{i i}^{(1)}\left(z_{2}\right)\right)+\frac{2}{\left|\operatorname{Im} z_{2}\right|^{2}} \mathbf{D}\left(G_{i i}^{(1)}\left(z_{1}\right)\right) \\
& =\left(\frac{1}{\left|\operatorname{Im} z_{1}\right|^{2}}+\frac{1}{\left|\operatorname{Im} z_{2}\right|^{2}}\right) O\left(\frac{1}{b_{n}}\right) .
\end{aligned}
$$

Therefore,

$$
\mathbf{D}\left(T_{2}\right) \leqslant \frac{\kappa_{4}^{2}}{b_{n}^{2}}\left(b_{n} O\left(\frac{1}{b_{n}}\right)+b_{n}^{2} O\left(\frac{1}{b_{n}}\right)\right)=O\left(\frac{1}{b_{n}}\right) .
$$

Now

$$
\begin{aligned}
\mathbf{D}\left(T_{3}\right) & \leqslant \frac{1}{b_{n}^{2}} \mathbf{D}\left(\widetilde{\gamma_{n-1}}\left(z_{1}\right) \widetilde{\gamma_{n-1}}\left(z_{2}\right)\right) \\
& \leqslant \frac{1}{b_{n}^{2}} \mathbb{E}\left[\left|\widetilde{\gamma_{n-1}}\left(z_{1}\right)\right|^{2}\left|\widetilde{\gamma_{n-1}}\left(z_{2}\right)\right|^{2}\right] \\
& \leqslant \frac{1}{b_{n}^{2}} \sqrt{\mathbb{E}\left[\left.\widetilde{\mid \widetilde{\gamma_{n-1}}}\left(z_{1}\right)\right|^{4}\right]} \sqrt{\mathbb{E}\left[\left|\widetilde{\gamma_{n-1}}\left(z_{2}\right)\right|^{4}\right]}=\frac{1}{b_{n}^{2}} O(1) .
\end{aligned}
$$

Last equality holds, since $\mathbb{E}\left[\left|\widetilde{\gamma_{n-1}}\left(z_{1}\right)\right|^{4}\right]=O(1)$. And finally

$$
\mathbf{D}\left(T_{1}\right)=\frac{4}{b_{n}^{2}} \mathbf{D}\left(\sum_{i, j \in I_{1}} G_{i j}^{(1)}\left(z_{1}\right) G_{i j}^{(1)}\left(z_{2}\right)\right) .
$$

Now using the Poincaré inequality

$$
\begin{aligned}
& \mathbf{D}\left(\sum_{i, j \in I_{1}} G_{i j}^{(1)}\left(z_{1}\right) G_{i j}^{(1)}\left(z_{2}\right)\right) \\
& \leqslant \frac{1}{m} \sum_{(s, t) \in I_{n}^{+}} \mathbb{E}\left[\left|\frac{\partial}{\partial w_{s t}} \sum_{i, j \in I_{1}} G_{i j}^{(1)}\left(z_{1}\right) G_{i j}^{(1)}\left(z_{2}\right)\right|^{2}\right] \\
& \quad \leqslant \frac{1}{m b_{n}} \sum_{(s, t) \in I_{n}^{+}} \mathbb{E}\left[\mid \sum_{i, j \in I_{1}} G_{i s}^{(1)}\left(z_{1}\right) G_{t j}^{(1)}\left(z_{1}\right) G_{i j}^{(1)}\left(z_{2}\right)\right.
\end{aligned}
$$




$$
\begin{gathered}
\left.+\left.G_{i j}^{(1)}\left(z_{1}\right) G_{i s}^{(1)}\left(z_{2}\right) G_{t j}^{(1)}\left(z_{2}\right)\right|^{2}\right] \\
\leqslant \frac{2}{m b_{n}} \sum_{(s, t) \in I_{n}^{+}} \mathbb{E}\left[\left|\sum_{i, j \in I_{1}} G_{i s}^{(1)}\left(z_{1}\right) G_{t j}^{(1)}\left(z_{1}\right) G_{i j}^{(1)}\left(z_{2}\right)\right|^{2}\right] \\
+\frac{2}{m b_{n}} \sum_{(s, t) \in I_{n}^{+}} \mathbb{E}\left[\left|\sum_{i, j \in I_{1}} G_{i j}^{(1)}\left(z_{1}\right) G_{i s}^{(1)}\left(z_{2}\right) G_{t j}^{(1)}\left(z_{2}\right)\right|^{2}\right] \\
=: I_{1}+I_{2} .
\end{gathered}
$$

We estimate

$$
\begin{aligned}
I_{1} & =\frac{2}{m b_{n}} \sum_{(s, t) \in I_{n}^{+}} \mathbb{E}\left[\left|\sum_{i, j \in I_{1}} G_{i s}^{(1)}\left(z_{1}\right) G_{t j}^{(1)}\left(z_{1}\right) G_{i j}^{(1)}\left(z_{2}\right)\right|^{2}\right] \\
& =\frac{2}{m b_{n}} \sum_{(s, t) \in I_{n}^{+}} \mathbb{E}\left[\left|\sum_{i \in I_{1}} G_{i s}^{(1)}\left(z_{1}\right) G_{t i}^{(1)}\left(z_{1}, z_{2}\right)\right|^{2}\right] \\
& =\frac{2}{m b_{n}} \sum_{(s, t) \in I_{n}^{+}} \mathbb{E}\left[\left|G_{s t}^{(1)}\left(z_{1}, z_{2}, z_{1}\right)\right|^{2}\right] \\
& \leqslant \frac{2}{m b_{n}} \mathbb{E}\left[\sum_{s, t=1}^{n}\left|G_{s t}^{(1)}\left(z_{1}, z_{2}, z_{1}\right)\right|^{2}\right]=\frac{2}{m b_{n}} \mathbb{E}\left[\|A\|_{F b}^{2}\right] \\
& =\frac{2}{m b_{n}} \mathbb{E}\left[\sum_{i=1}^{n} \beta_{i}^{2}\right] \leqslant \frac{C\left(z_{1}, z_{2}\right)}{m b_{n}} O\left(b_{n}\right)=O(1),
\end{aligned}
$$

where $\|\cdot\|_{F b}$ is the Frobenius norm, $\beta_{i}$ are the eigenvalues of $V V^{*}$, and $V$ is the following matrix:

$$
\begin{aligned}
V_{n \times n}= & {\left[\begin{array}{cccc}
G_{11}^{(1)}\left(z_{1}\right) & G_{12}^{(1)}\left(z_{1}\right) & \cdots & G_{1 k_{n}}^{(1)}\left(z_{1}\right) \\
G_{21}^{(1)}\left(z_{1}\right) & G_{22}^{(1)}\left(z_{1}\right) & \cdots & G_{2 k_{n}}^{(1)}\left(z_{1}\right) \\
\vdots & \vdots & & \vdots \\
G_{n 1}^{(1)}\left(z_{1}\right) & G_{n 2}^{(1)}\left(z_{1}\right) & \cdots & G_{n k_{n}}^{(1)}\left(z_{1}\right)
\end{array}\right]_{n \times k_{n}} } \\
& \times\left[\begin{array}{cccc}
G_{11}^{(1)}\left(z_{2}\right) & G_{12}^{(1)}\left(z_{2}\right) & \cdots & G_{1 k_{n}}^{(1)}\left(z_{2}\right) \\
G_{21}^{(1)}\left(z_{2}\right) & G_{22}^{(1)}\left(z_{2}\right) & \cdots & G_{2 k_{n}}^{(1)}\left(z_{2}\right) \\
\vdots & \vdots & & \vdots \\
G_{k_{n} 1}^{(1)}\left(z_{2}\right) & G_{k_{n} 2}^{(1)}\left(z_{2}\right) & \cdots & G_{k_{n} k_{n}}^{(1)}\left(z_{2}\right)
\end{array}\right]_{k_{n} \times k_{n}} \\
& \times\left[\begin{array}{cccc}
G_{11}^{(1)}\left(z_{1}\right) & G_{12}^{(1)}\left(z_{1}\right) & \cdots & G_{1 n}^{(1)}\left(z_{1}\right) \\
G_{21}^{(1)}\left(z_{1}\right) & G_{22}^{(1)}\left(z_{1}\right) & \cdots & G_{2 n}^{(1)}\left(z_{1}\right) \\
\vdots & \vdots & & \vdots \\
G_{k_{n} 1}^{(1)}\left(z_{1}\right) & G_{k_{n} 2}^{(1)}\left(z_{1}\right) & \cdots & G_{k_{n} n}^{(1)}\left(z_{1}\right)
\end{array}\right]_{k_{n} \times n}
\end{aligned}
$$


Here we denoted the elements of set $I_{1}$ as $I_{1}=\left\{1,2, \ldots, k_{n}\right\}$. Observe that $k_{n}=2 b_{n}$. Rank of $V \leqslant k_{n}=O\left(b_{n}\right)$. This implies

$$
\sum_{i=1}^{n} \beta_{i}^{2} \leqslant k_{n} C\left(z_{1}, z_{2}\right)=O\left(b_{n}\right) C\left(z_{1}, z_{2}\right) .
$$

Therefore, $\mathbf{D}\left(T_{1}\right)=O\left(1 / b_{n}^{2}\right)$, and hence $\mathbf{D}\left\{b_{n} \mathbb{E}_{1}\left[A^{\circ}\left(z_{1}\right) A^{\circ}\left(z_{2}\right)\right]\right\}=O\left(1 / b_{n}\right)$.

Second part of (iii) follows from the following two facts with the help of Cauchy's theorem:

$$
\begin{gathered}
b_{n} \mathbb{E}_{1}\left[A^{\circ}\left(z_{1}\right) B^{\circ}\left(z_{2}\right)\right]=b_{n} \frac{d}{d z_{2}} \mathbb{E}_{1}\left[A^{\circ}\left(z_{1}\right) A^{\circ}\left(z_{2}\right)\right], \\
\mathbf{D}\left\{b_{n} \mathbb{E}_{1}\left\{A^{\circ}\left(z_{1}\right) A^{\circ}\left(z_{2}\right)\right\}\right\}=O\left(\frac{1}{b_{n}}\right) .
\end{gathered}
$$

Here we skip the details.

P r o o f of (i v). Using (25) and (30), and proceeding as in the proof of Proposition 2,

$$
\begin{aligned}
\mathbb{E}\left[\left|\gamma_{n-1}^{\circ}(z)-\gamma_{n}^{\circ}(z)\right|^{4}\right]= & \mathbb{E}\left[\mid\left(\operatorname{Tr} G^{(1)}(z)-\mathbb{E}\left[\operatorname{Tr} G^{(1)}(z)\right]\right)\right. \\
& \left.-\left.(\operatorname{Tr} G(z)-\mathbb{E}[\operatorname{Tr} G(z)])\right|^{4}\right] \\
= & \mathbb{E}\left[\left|\frac{1+B(z)}{A(z)}-\mathbb{E}\left[\frac{1+B(z)}{A(z)}\right]\right|^{4}\right] \\
& \leqslant \frac{C}{|\operatorname{Im} z|^{8}}\left(\mathbb{E}\left[\left|A^{\circ}\right|^{4}\right]+\mathbb{E}\left[\left|B^{\circ}\right|^{4}\right]+\mathbb{E}\left[\left|A^{\circ}\right|^{4}\right]\right) \\
= & O\left(\frac{1}{b_{n}^{2}}\right) .
\end{aligned}
$$

The last equality follows from the estimates (37) and (38).

Using martingale differences as in the proof of Proposition 2,

$$
\mathbb{E}\left[\left|\gamma_{n}^{\circ}\right|^{4}\right] \leqslant C n \sum_{k=1}^{n} \mathbb{E}\left[\left|\gamma_{n}-\mathbb{E}_{k}\left[\gamma_{n}\right]\right|^{4}\right] .
$$

Consider for $k=1$, others will be similar.

$$
\begin{aligned}
\mathbb{E}\left[\left|\gamma_{n}-\mathbb{E}_{1}\left[\gamma_{n}\right]\right|^{4}\right] & =\mathbb{E}\left[\left|\operatorname{Tr}\left(G-G^{(1)}\right)-\mathbb{E}_{1}\left[\operatorname{Tr}\left(G-G^{(1)}\right)\right]\right|^{4}\right] \\
& =\mathbb{E}\left[\left|\frac{1+B(z)}{A(z)}-\mathbb{E}_{1}\left[\frac{1+B(z)}{A(z)}\right]\right|^{4}\right] \\
& \leqslant C_{1}(z) \mathbb{E}\left[\left|A_{1}^{\circ}\right|^{4}\right]+C_{2}(z) \mathbb{E}\left[\left|B_{1}^{\circ}\right|^{4}\right]=O\left(\frac{1}{b_{n}^{2}}\right) .
\end{aligned}
$$

The last equality follows from (51) and (52). Hence we have the result. 
P r o o f of (v). Using resolvent identity

$$
\left(X_{2}-z I\right)^{-1}=\left(X_{1}-z I\right)^{-1}+\left(X_{1}-z I\right)^{-1}\left(X_{1}-X_{2}\right)\left(X_{2}-z I\right)^{-1},
$$

we have

$$
z G_{11}(z)=-1+\sum_{(1, k) \in I_{n}} m_{1 k} G_{k 1},
$$

where $I_{n}$ is defined in (1) and $m_{i j}$ 's are defined in (2). Now to analyse the terms $\mathbb{E}\left[m_{1 k} G_{k 1}\right]$, we use the following (see, e.g., [10]): Given $\xi$, a real-valued random variable with $p+2$ finite moments, and $\phi$, a function from $\mathbb{C}$ to $\mathbb{R}$ with $p+1$ continuous and bounded derivatives then:

$$
\mathbb{E}[\xi \phi(\xi)]=\sum_{a=0}^{p} \frac{\kappa_{a+1}}{a !} \mathbb{E}\left[\phi^{(a)}(\xi)\right]+\epsilon_{p+1},
$$

where $\kappa_{a}$ is the $a$-th cumulant of $\xi,\left|\epsilon_{p+1}\right| \leqslant C \sup _{t}\left|\phi^{(p+1)}(t)\right| \mathbb{E}\left[|\xi|^{p+2}\right]$ and $C$ depends only on $p$. Since $f_{n}(z)=(1 / n) \mathbb{E}[\operatorname{Tr} G(z)]=\mathbb{E}\left[G_{11}(z)\right]$, using (53) and (54) we get

$$
\begin{aligned}
z f_{n}(z) & =-1+\sum_{(1, k) \in I_{n}} \mathbb{E}\left[m_{1 k} G_{k 1}\right] \\
& =-1-\sum_{k \in I_{1}} \frac{1}{b_{n}} \mathbb{E}\left[G_{k 1}^{2}+G_{k k} G_{11}\right]+r_{n},
\end{aligned}
$$

where $r_{n}$ contains the third cumulant term corresponding to $p=2$ in (54) for $k \neq 1$, and the error terms due to the truncation of the decoupling formula (54) at $p=2$ for $k \neq 1$ and at $p=0$ for $k=1$. We write (55)

$$
\begin{aligned}
z f_{n}(z)= & -1-\frac{1}{b_{n}} \mathbb{E}\left[G_{11}\right] \mathbb{E}\left[\sum_{k \in I_{1}} G_{k k}\right]-\frac{1}{b_{n}} \operatorname{Cov}\left(G_{11}, \sum_{k \in I_{1}} G_{k k}\right) \\
& -\frac{1}{b_{n}} \mathbb{E}\left[\sum_{k \in I_{1}} G_{k 1}^{2}\right]+r_{n} \\
= & -1-f_{n}(z)\left(2 f_{n}(z)\right)-\frac{1}{b_{n}} \operatorname{Cov}\left(G_{11}, \sum_{k \in I_{1}} G_{k k}\right) \\
& -\frac{1}{b_{n}} \mathbb{E}\left[\sum_{k \in I_{1}} G_{k 1}^{2}\right]+r_{n} .
\end{aligned}
$$

Now, by the Cauchy-Schwarz inequality and (47) we get

$$
\begin{aligned}
\frac{1}{b_{n}}\left|\operatorname{Cov}\left(G_{11}, \sum_{k \in I_{1}} G_{k k}\right)\right| & \leqslant \frac{1}{b_{n}} \sqrt{\mathbf{D}\left(G_{11}\right)} \sqrt{\mathbf{D}\left(\sum_{k \in I_{1}} G_{k k}\right)} \\
& \leqslant \frac{1}{b_{n}} 2|\operatorname{Im} z|^{-1} \sqrt{O\left(|\operatorname{Im} z|^{-4}\right)} \\
& =O\left(\frac{1}{b_{n}|\operatorname{Im} z|^{3}}\right) .
\end{aligned}
$$


Also notice that

$$
\frac{1}{b_{n}}\left|\mathbb{E}\left(\sum_{k \in I_{1}} G_{k 1}^{2}\right)\right| \leqslant \frac{1}{b_{n}}|\operatorname{Im} z|^{-2} .
$$

We claim $r_{n}=O\left(1 /\left(b_{n}|\operatorname{Im} z|^{4}\right)\right)$. To prove this, observe that the third cumulant term gives

$$
\frac{\kappa_{3}}{2 b_{n}^{3 / 2}} \mathbb{E}\left[\sum_{k \in I_{1}} 2\left(G_{1 k}\right)^{3}+6 G_{11} G_{1 k} G_{k k}\right] .
$$

Since $\sum_{k \in I_{1}}\left|G_{1 k}\right|^{2} \leqslant\|G\|^{2} \leqslant|\operatorname{Im} z|^{-2}$ and $\left|G_{i j}\right| \leqslant|\operatorname{Im} z|^{-1}$, we conclude that the third cumulant term contributes $O\left(1 /\left(b_{n}|\operatorname{Im} z|^{3}\right)\right)$ to $r_{n}$. In a similar manner, the error due to truncation of decoupling formula (54) at $p=2$ is $O\left(1 /\left(b_{n}|\operatorname{Im} z|^{4}\right)\right)$. Similarly, the error term due to truncation of decoupling formula at $p=0$ for $k=1$ is $O\left(1 /\left(b_{n}|\operatorname{Im} z|^{2}\right)\right)$. Thus the claim is proved. Hence

$$
z f_{n}(z)=-1-2 f_{n}^{2}(z)+O\left(\frac{1}{b_{n}|\operatorname{Im} z|^{4}}\right) \quad \text { for } z \in \mathbb{C} \backslash \mathbb{R} .
$$

Now following similar argument given in the proof of (3.1) in [14], one can show that

$$
\left|f_{n}(z)-f(z)\right| \leqslant O\left(\frac{1}{b_{n}|\operatorname{Im} z|^{6}}\right)
$$

where $f(z)=\left(-z+\sqrt{z^{2}-8}\right) / 4$.

P r o o f of (vi). Recall $A(z)=z-b_{n}^{-1 / 2} w_{11}+b_{n}^{-1} \sum_{i, j \in I_{1}} G_{i j}^{(1)} w_{1 i} w_{1 j}$. Now using (41) with $G$ replaced by $G^{(1)}$, we have

$$
\begin{aligned}
(\mathbb{E}[A(z)])^{-1} & =\frac{1}{z+b_{n}^{-1} \sum_{j \in I_{1}} \mathbb{E}\left[G_{j j}^{(1)}\right]}=\frac{1}{z+2 f_{n}(z)} \\
& =(z+2 f(z))^{-1}+O\left(b_{n}^{-1}\right)=-f(z)+O\left(b_{n}^{-1}\right) .
\end{aligned}
$$

Hence $(\mathbb{E}[A(z)])^{-1}=-f(z)+O\left(b_{n}^{-1}\right)$. To prove the second part, observe that

$$
\begin{aligned}
\mathbb{E}[B(z)] & =\frac{1}{b_{n}} \mathbb{E}\left[\sum_{i, j \in I_{1}}\left(G^{(1)} G^{(1)}\right)_{i j} w_{1 i} w_{1 j}\right]=\frac{1}{b_{n}} \mathbb{E}\left[\sum_{i \in I_{1}}\left(G^{(1)} G^{(1)}\right)_{i i}\right] \\
& =\frac{1}{b_{n}} \mathbb{E}\left[\sum_{i \in I_{1}} \sum_{k=2}^{n} G_{i k}^{(1)} G_{k i}^{(1)}\right]=\frac{1}{b_{n}} \sum_{i \in I_{1}} \frac{d}{d z} G_{i i}^{(1)} .
\end{aligned}
$$

Again using (41) and Cauchy's integral formula, we have

$$
\mathbb{E}[B(z)]=\frac{d}{d z}\left(2 f_{n}(z)\right)=2 f^{\prime}(z)+O\left(b_{n}^{-1}\right) .
$$

This completes the proof of Lemma 2. 


\subsection{Proof of (29).}

$\mathrm{P}$ r o o f. We have to find the limit of

$$
\mathbb{E}\left[T_{n}\right]=\frac{2}{b_{n}} \mathbb{E}\left[\sum_{i, j \in I_{1}} G_{i j}^{(1)}(z) G_{i j}^{(1)}\left(z_{\mu}\right)\right]
$$

as $n \rightarrow \infty$, where $I_{1}=\left\{2 \leqslant i \leqslant n:(1, i) \in I_{n}\right\}$. Let $f, g \in C_{b}(\mathbb{R})$. Define a bilinear form on $C_{b}(\mathbb{R})$ as

$$
\langle f, g\rangle_{n}=\frac{1}{b_{n}} \mathbb{E}\left[\sum_{i, j \in I_{1}} f(M)_{i j} g(M)_{j i}\right] .
$$

Then $\mathbb{E}\left[T_{n}\right]=\left\langle h(M), h_{\mu}(M)\right\rangle_{n}$, where $h(x)=(x-z)^{-1}$ and $h_{\mu}(x)=(x-$ $\left.z_{\mu}\right)^{-1}$.

Lemma 3. For $f, g \in C_{b}(\mathbb{R})$ the limit $\langle f, g\rangle=\lim _{n \rightarrow \infty}\langle f, g\rangle_{n}$ exists.

$\mathrm{P}$ r o o f. The idea of the proof is similar to the proof of Lemma 3.11 of [9]. First we prove this result for monomials. Although monomials are unbounded, still (57) makes sense for all $n$, since all moments of the entries of $M$ are finite. Consider $f(x)=x^{l}$, and $g(x)=x^{m}$ where $l, m \in \mathbb{N}$. Then

$$
\left\langle x^{l}, x^{m}\right\rangle_{n}=\frac{1}{b_{n}^{1+(l+m) / 2}} \sum_{\substack{\left(i_{0}, i_{1}\right),\left(i_{1}, i_{2}\right), \ldots,\left(i_{l+m-1}, i_{0}\right) \in I_{n} \\ i_{0}, i_{l} \in I_{1}}} \mathbb{E}\left[w_{i_{0} i_{1}} w_{i_{1} i_{2}} \cdots w_{i_{l+m-1} i_{0}}\right] .
$$

If $(l+m)$ is odd, then $\left\langle x^{l}, x^{m}\right\rangle_{n} \rightarrow 0$ using independence of matrix entries and $\mathbb{E}\left[w_{i j}\right]=0$, and order counting of independent vertices. The argument is similar to the combinatorial argument given in the proof of Wigner semicircular law (see [1]). We leave it for the reader.

Now we assume $l+m$ is even. Then

$$
\begin{aligned}
\left\langle x^{l},\right. & \left.x^{m}\right\rangle_{n}=\frac{1}{b_{n}^{1+(l+m) / 2}} \sum_{\substack{\left(i_{0}, i_{1}\right),\left(i_{1}, i_{2}\right), \ldots,\left(i_{l}+m-1 \\
i_{0}, i_{l} \in I_{1}\right.}} \mathbb{E}\left[w_{i_{0} i_{1}} w_{i_{1} i_{2}} \cdots w_{i_{l+m-1} i_{0}}\right] \\
= & \frac{1}{b_{n}^{1+(l+m) / 2}} \\
& \times \sum_{\substack{\left(i_{0}, i_{1}\right),\left(i_{1}, i_{2}\right), \ldots, I_{n} \\
i_{0}, i_{l} \in I_{1}}} \mathbb{E}\left[w_{1 i_{0}} w_{i_{0} i_{1}} w_{i_{1} i_{2}} \cdots w_{i_{l+m-1} i_{0}} w_{i_{0} 1}\right]+O\left(b_{n}^{-1}\right) \\
= & \frac{1}{b_{n}^{1+(l+m) / 2}} \sum_{\substack{\left.\left(i_{0}, i_{1}\right),\left(i_{1}, i_{2}\right), \ldots,\left(i_{l}\right) \in I_{n} \\
\left(1, i_{0}\right),\left(1, i_{l}\right) \in I_{n}-1, i_{0}\right) \in I_{n}}} \mathbb{E}\left[w_{1 i_{0}} w_{i_{0} i_{1}} w_{i_{1} i_{2}} \cdots w_{i_{l+m-1} i_{0}} w_{i_{0} 1}\right]+O\left(b_{n}^{-1}\right) .
\end{aligned}
$$


The second last equality in (58) holds due to order calculation of independent vertices and independence of matrix entries. Now define for $k=1,2, \ldots, l+$ $m$,

$$
\begin{aligned}
& x_{k}=\left\{\begin{array}{ll}
i_{k}-i_{k-1} & \text { if }\left|i_{k}-i_{k-1}\right| \leqslant b_{n}, \\
\left(i_{k}-i_{k-1}\right)-n & \text { if } i_{k}-i_{k-1}>b_{n}, \\
n+\left(i_{k}-i_{k-1}\right) & \text { if } i_{k}-i_{k-1}<-b_{n}
\end{array} \quad \text { with } i_{l+m}=i_{0},\right. \\
& x_{0}= \begin{cases}i_{0}-1 & \text { if }\left|i_{0}-1\right| \leqslant b_{n} \\
\left(i_{0}-1\right)-n & \text { if } i_{0}-1>b_{n}\end{cases} \\
& x_{l+m+1}= \begin{cases}1-i_{0} & \text { if }\left|1-i_{0}\right| \leqslant b_{n} \\
n+\left(1-i_{0}\right) & \text { if } 1-i_{0}<-b_{n} .\end{cases}
\end{aligned}
$$

Note, $x_{0}=-x_{l+m+1}$. Since $l, m$ are fixed and $b_{n} \rightarrow \infty$, for large $n$ the restrictions $\left\{\left(i_{0}, i_{1}\right),\left(i_{1}, i_{2}\right), \ldots,\left(i_{l+m-1}, i_{0}\right) \in I_{n}\right.$ and $\left.\left(1, i_{0}\right),\left(1, i_{l}\right) \in I_{n}\right\}$ are equivalent to $\left\{\left|x_{0}\right|,\left|x_{1}\right|, \ldots,\left|x_{l+m}\right| \leqslant b_{n}, x_{0}+x_{1}+\cdots+x_{l+m}+x_{l+m+1}=0\right.$ and $\left.\left|x_{0}+x_{1}+\cdots+x_{l}\right| \leqslant b_{n}\right\}$. Also observe that $x_{0}+x_{1}+\cdots+x_{l+m}+x_{l+m+1}=0$ is same as $x_{1}+\cdots+x_{l+m}=0$ since $x_{0}=-x_{l+m+1}$. Therefore for large $n$

$$
\begin{aligned}
& \left\langle x^{l}, x^{m}\right\rangle_{n}=\frac{1}{\sum_{\substack{x_{1}+\cdots+x_{l+m}=0 \\
\left|x_{i}\right| \leqslant b_{n}, 0 \leqslant i \leqslant l+m,\left|x_{0}+x_{1}+\cdots+x_{l}\right| \leqslant b_{n}}}^{1+(l+m) / 2}} \mathbb{E}\left[w_{1 i_{0}} w_{i_{0} i_{1}} w_{i_{1} i_{2}} \cdots w_{i_{l+m-1} i_{0}} w_{i_{0} 1}\right)+O\left(b_{n}^{-1}\right) .
\end{aligned}
$$

Without loss of generality, we assume that $l \leqslant m$. Each $\left\{i_{0}, i_{1}, i_{2}, \ldots\right.$, $\left.i_{l+m-1}, i_{0}\right\}$ is a closed path such that distance between the end points of each edge is bounded by $b_{n}$. As in the proof of Wigner semicircular law only the paths whose edges are pair matched contributes to the limit, here also, only such paths contribute to the limit. And contribution of each path is $\mathbb{E}\left\{w_{1 i_{0}} w_{i_{0} i_{1}} \cdots w_{i_{l+m-1} i_{0}} w_{i_{0} 1}\right\}=1$ since $\mathbb{E}\left\{w_{i j}^{2}\right\}=1$. Each such path corresponds to a Dyck path of length $(l+m)$. Recall that a Dyck path $(S(0), S(1), \ldots, S(l+m))$ of length $(l+m)$ satisfies (see [1])

$$
\begin{gathered}
S(0)=S(l+m)=0, \quad S(1), S(2), \ldots, S(l+m-1) \geqslant 0, \\
|S(i+1)-S(i)|=1, \quad \text { for } i=0,1, \ldots, l+m-1 .
\end{gathered}
$$

Specifically, $S(t+1)-S(t)=1$ if the non oriented edge $\left(i_{t}, i_{t+1}\right)$ appears in $\left\{i_{0}, i_{1}, \ldots, i_{l+m-1}, i_{0}\right\}$ for the first time and $S(t+1)-S(t)=-1$ if the edge $\left(i_{t}, i_{t+1}\right)$ appears in $\left\{i_{0}, i_{1}, \ldots, i_{l+m-1}, i_{0}\right\}$ for the second time.

Here each Dyck path does not give equal contribution to the limit due to the condition that $\left(1, i_{l}\right) \in I_{n}$ and in terms of $x_{i}$, which is same as $\mid x_{0}+x_{1}+$ $\cdots+x_{l} \mid \leqslant b_{n}$. We have to take into account this condition. Suppose $S(l)=k$, $0 \leqslant k \leqslant l$. Then during the first $l$ steps of the path $\left\{i_{0}, i_{1}, \ldots, i_{l+m-1}, i_{0}\right\}, k$ edges appear only once and $(l-k) / 2$ edges appear twice. The edges appearing 
twice, the corresponding two number $x_{i}$ have same absolute value but with different sign. We rename the remaining $k$ numbers $x_{i}$ which appear only once as $y_{1}, y_{2}, \ldots, y_{k}$ (according to their order of appearance) and $x_{0}$ as $y_{0}$. So the condition $\left|x_{0}+x_{1}+\cdots+x_{l}\right| \leqslant b_{n}$ reduces to $\left|y_{0}+y_{1}+\cdots+y_{k}\right| \leqslant b_{n}$. Therefore,

$$
\begin{aligned}
\left\langle x^{l}, x^{m}\right\rangle_{n}= & \frac{1}{b_{n}^{1+(l+m) / 2}} \sum_{k=0}^{l} \#\{\text { Dyck path of length } l+m \text { with } S(l)=k\} \\
& \times \#\left\{\left|y_{0}\right| \leqslant b_{n},\left|y_{1}\right| \leqslant b_{n}, \ldots,\left|y_{k}\right| \leqslant b_{n}, \ldots,\left|y_{l+m}\right| \leqslant b_{n},\right. \\
& \left.\left|y_{0}+y_{1}+\cdots+y_{k}\right| \leqslant b_{n}\right\}+O\left(b_{n}^{-1}\right)
\end{aligned}
$$

and

$$
\begin{aligned}
& \left\langle x^{l}, x^{m}\right\rangle=\lim _{n \rightarrow \infty}\left\langle x^{l}, x^{m}\right\rangle_{n} \\
& =(\sqrt{2})^{l+m+2} \sum_{k=0}^{l} \#\{\text { Dyck path of length } l+m \text { with } S(l)=k\} \\
& \quad \times \operatorname{Vol}\left\{\left|t_{0}\right| \leqslant \frac{1}{2},\left|t_{1}\right| \leqslant \frac{1}{2}, \ldots,\left|t_{(l+m) / 2}\right| \leqslant \frac{1}{2},\left|t_{0}+t_{1}+\cdots+t_{k}\right| \leqslant \frac{1}{2}\right\} \\
& =(\sqrt{2})^{l+m+2} \sum_{k=0}^{l} \#\{\text { Dyck path of length } l+m \text { with } S(l)=k\} \\
& \quad \times P\left(\left|T_{0}+T_{1}+\cdots+T_{k}\right| \leqslant \frac{1}{2}\right),
\end{aligned}
$$

where $T_{0}, T_{1}, \ldots, T_{(l+m) / 2}$ are independent random variables uniformly distributed on $[-1 / 2,1 / 2]$. Let $S_{k+1}=T_{0}+T_{1}+\cdots+T_{k}$. Then

$$
\mathbb{E}\left[e^{i x S_{k+1}}\right]=\left(\mathbb{E}\left[e^{i x T_{0}}\right]\right)^{k+1}=\left(\frac{\sin x / 2}{x / 2}\right)^{k+1}
$$

Using inversion formula, the density of $S_{k+1}$ is given by

$$
f_{k+1}(s)=\frac{1}{2 \pi} \int_{-\infty}^{\infty} e^{-i x s}\left(\frac{\sin x / 2}{x / 2}\right)^{k+1} d x .
$$

Now

$$
\begin{aligned}
\gamma_{k+1} & :=P\left(\left|S_{k+1}\right| \leqslant \frac{1}{2}\right)=\int_{-1 / 2}^{1 / 2} f_{k+1}(s) d s \\
& =\frac{1}{2 \pi} \int_{-\infty}^{\infty}\left(\frac{\sin x / 2}{x / 2}\right)^{k+2} d x=f_{k+2}(0),
\end{aligned}
$$


using [5] we get exact formula of $\gamma_{k+1}$ :

$$
\gamma_{k+1}= \begin{cases}\frac{1}{(k+1) !} \sum_{s=0}^{(k+1) / 2}(-1)^{s}\left(\begin{array}{c}
k+2 \\
s
\end{array}\right)\left(\frac{k+1}{2}-s+\frac{1}{2}\right)^{k+1} & \text { if } k+1 \text { even }, \\
\frac{1}{(k+1) !} \sum_{s=0}^{k / 2}(-1)^{s}\left(\begin{array}{c}
k+2 \\
s
\end{array}\right)\left(\frac{k+1}{2}-s+\frac{1}{2}\right)^{k+1} & \text { if } k+1 \text { odd } .\end{cases}
$$

The number of Dyck path of length $l+m$ with $S(l)=k$ is

$$
\begin{gathered}
{\left[\left(\begin{array}{c}
l \\
\frac{l-k}{2}
\end{array}\right)-\left(\begin{array}{c}
l \\
\frac{l-k-2}{2}
\end{array}\right)\right] \times\left[\left(\begin{array}{c}
m \\
\frac{m-k}{2}
\end{array}\right)-\left(\begin{array}{c}
m \\
\frac{m-k-2}{2}
\end{array}\right)\right]} \\
=\frac{(k+1)^{2}}{(l+1)(m+1)}\left(\begin{array}{l}
l+1 \\
\frac{l+k+2}{2}
\end{array}\right)\left(\begin{array}{l}
m+1 \\
\frac{m+k+2}{2}
\end{array}\right) .
\end{gathered}
$$

Hence from (59) and (60), we get

$$
\left\langle x^{l}, x^{m}\right\rangle=(\sqrt{2})^{l+m+2} C_{l, m},
$$

where $C_{l, m}=0$ if $(l+m)$ is odd and

$$
\begin{aligned}
C_{l, m} & =\sum_{k=0}^{l} \frac{(k+1)^{2}}{(l+1)(m+1)}\left(\begin{array}{c}
l+1 \\
\frac{l+k+2}{2}
\end{array}\right)\left(\begin{array}{c}
m+1 \\
\frac{m+k+2}{2}
\end{array}\right) \gamma_{k+1} \\
& = \begin{cases}\sum_{k=0}^{l / 2} \frac{(2 k+1)^{2}}{(l+1)(m+1)}\left(\begin{array}{c}
l+1 \\
\frac{l-2 k}{2}
\end{array}\right)\left(\begin{array}{c}
m+1 \\
\frac{m-2 k}{2}
\end{array}\right) \gamma_{2 k+1} & \text { if } l \text { even, } \\
\sum_{k=0}^{l-1) / 2} \frac{(2 k+2)^{2}}{(l+1)(m+1)}\left(\begin{array}{c}
l+1 \\
\frac{l-2 k-1}{2}
\end{array}\right)\left(\begin{array}{c}
m+1 \\
\frac{m-2 k-1}{2}
\end{array}\right) \gamma_{2 k+2} & \text { if } l \text { odd },\end{cases}
\end{aligned}
$$

if $(l+m)$ is even and $l \leqslant m$, otherwise, $C_{l, m}=C_{m, l}$. If $f, g$ are polynomials, $f(x)=\sum_{i=0}^{p} a_{i} x^{i}, g(x)=\sum_{i=0}^{q} b_{i} x^{i}$, then by linearity

$$
\langle f, g\rangle=\sum_{i=0}^{p} \sum_{j=0}^{q} a_{i} b_{j}(\sqrt{2})^{i+j+2} C_{i, j} .
$$

For general bounded continuous functions $f, g$, to show that $\langle f, g\rangle$ exists we have to use the Stone-Weierstrass theorem to approximate $f, g$ by appropriate polynomial and then (61). The argument is similar to the argument given in the proof of Lemma 3.11 of [9]. We skip the details.

In the next lemma we diagonalize the bilinear form $\langle f, g\rangle$.

Lemma 4. Let $\left\{U_{n}(x)\right\}_{n \geqslant 0}$ be the rescaled Chebyshev polynomial of the second kind on $[-2 \sqrt{2}, 2 \sqrt{2}]$,

$$
U_{n}(x)=\sum_{k=0}^{\lfloor n / 2\rfloor}(-1)^{k}\left(\begin{array}{c}
n-k \\
k
\end{array}\right)\left(\frac{x}{\sqrt{2}}\right)^{n-2 k} .
$$


Then $\left\{U_{n}(x)\right\}$ are orthogonal with respect to the bilinear form (61), that is,

$$
\left\langle U_{n}, U_{m}\right\rangle=2 \delta_{n m} \gamma_{n+1},
$$

where $\gamma_{n+1}$ is defined in (59).

$\mathrm{P} \mathrm{r}$ o o $\mathrm{f}$. The proof of this lemma is similar to the proof of Lemma 3.12 of [9]. For sake of completeness we outline it here. Since $\left\langle x^{l}, x^{m}\right\rangle=0$ if $l+m$ is odd, from linearity $\left\langle U_{l}, U_{m}\right\rangle=0$ if $l+m$ is odd. We are left to compute $\left\langle U_{2 n}, U_{2 m}\right\rangle$ and $\left\langle U_{2 n+1}, U_{2 m+1}\right\rangle$. We first compute $\left\langle x^{2 l}, U_{2 n}\right\rangle$ and $\left\langle x^{2 l+1}, U_{2 n+1}\right\rangle$ for $l=0,1, \ldots, n$ :

$$
\begin{aligned}
& \left\langle x^{2 l}, U_{2 n}\right\rangle=(\sqrt{2})^{2 l+2} \sum_{k=0}^{n}(-1)^{k}\left(\begin{array}{c}
2 n-k \\
k
\end{array}\right) C_{2 l, 2 n-2 k} \\
& =(\sqrt{2})^{2 l+2}\left[\sum_{k=0}^{n-l}(-1)^{k}\left(\begin{array}{c}
2 n-k \\
k
\end{array}\right) \sum_{t=0}^{l} \frac{(2 t+1)^{2}}{(2 l+1)(2 n-2 k+1)}\right. \\
& \times\left(\begin{array}{c}
2 l+1 \\
l-t
\end{array}\right)\left(\begin{array}{c}
2 n-2 k+1 \\
n-k-t
\end{array}\right) \gamma_{2 t+1} \\
& +\sum_{k=n-l+1}^{n}(-1)^{k}\left(\begin{array}{c}
2 n-k \\
k
\end{array}\right) \sum_{t=0}^{n-k} \frac{(2 t+1)^{2}}{(2 l+1)(2 n-2 k+1)} \\
& \left.\times\left(\begin{array}{c}
2 l+1 \\
l-t
\end{array}\right)\left(\begin{array}{c}
2 n-2 k+1 \\
n-k-t
\end{array}\right) \gamma_{2 t+1}\right] \\
& =(\sqrt{2})^{2 l+2} \sum_{t=0}^{l} \frac{(2 t+1)^{2}}{2 l+1}\left(\begin{array}{c}
2 l+1 \\
l-t
\end{array}\right) \\
& \times\left[\sum_{k=0}^{n-t} \frac{(-1)^{k}(2 n-k) !}{k !(n-k-t) !(n-k+t+1) !}\right] \gamma_{2 t+1} \\
& =(\sqrt{2})^{2 l+2} \sum_{t=0}^{l} \frac{(2 t+1)^{2}}{2 l+1}\left(\begin{array}{c}
2 l+1 \\
l-t
\end{array}\right) G_{1}(n, t) \gamma_{2 t+1},
\end{aligned}
$$

where

$$
G_{1}(n, t)=\sum_{k=0}^{n-t} \frac{(-1)^{k}(2 n-k) !}{k !(n-k-t) !(n-k+t+1) !} .
$$

Similarly,

$$
\begin{aligned}
\left\langle x^{2 l+1}, U_{2 n+1}\right\rangle= & (\sqrt{2})^{2 l+3} \sum_{t=0}^{l} \frac{(2 t+2)^{2}}{2 l+2}\left(\begin{array}{c}
2 l+2 \\
l-t
\end{array}\right) \\
& \times\left[\sum_{k=0}^{n-t} \frac{(-1)^{k}(2 n+1-k) !}{k !(n-k-t) !(n-k+t+2) !}\right] \gamma_{2 t+2} \\
= & (\sqrt{2})^{2 l+3} \sum_{t=0}^{l} \frac{(2 t+2)^{2}}{2 l+2}\left(\begin{array}{c}
2 l+2 \\
l-t
\end{array}\right) G_{2}(n, t) \gamma_{2 t+2},
\end{aligned}
$$


where

$$
G_{2}(n, t)=\sum_{k=0}^{n-t} \frac{(-1)^{k}(2 n+1-k) !}{k !(n-k-t) !(n-k+t+2) !} .
$$

$G_{1}(n, t)$ and $G_{2}(n, t)$ can be written in terms of hypergeometric function as follows:

$$
\begin{aligned}
& G_{1}(n, t)=\frac{(2 n) !}{(n-t) !(n+t+1) !}{ }_{2} F_{1}\left(\begin{array}{c}
-(n-t),-(n+t+1) \\
-2 n
\end{array} \quad 1\right), \\
& G_{2}(n, t)=\frac{(2 n+1) !}{(n-t) !(n+t+2) !}{ }_{2} F_{1}\left(\begin{array}{c}
-(n-t),-(n+t+2) \\
-2 n-1
\end{array}\right),
\end{aligned}
$$

where ${ }_{2} F_{1}$ is a hypergeometric function. By the Chu-Vandermonde identity (see $[2])$, we have

$$
\begin{aligned}
& { }_{2} F_{1}\left(\begin{array}{c}
-(n-t),-(n+t+1) \\
-2 n
\end{array} \begin{array}{c}
1 \\
{ }_{2} F_{1}\left(\begin{array}{c}
-(n-t),-(n+t+2) \\
-2 n-1
\end{array}\right.
\end{array} \begin{array}{c}
(-2 n)_{n-t} \\
-1
\end{array}\right)=\frac{(-n+t+1)_{n-t}}{(-2 n-1)_{n-t}},
\end{aligned}
$$

where $(a)_{n}=a(a+1) \cdots(a+n-1)$. Since

$$
(-n+t+1)_{n-t}= \begin{cases}0 & \text { if } t=0,1, \ldots, n-1 \\ 1 & \text { if } t=n\end{cases}
$$

we have $G_{1}(n, t)=0, G_{2}(n, t)=0$ for $t=0,1, \ldots, n-1$ and $G_{1}(n, n)=$ $1 /(2 n+1), G_{2}(n, n)=1 /(2 n+2)$. Therefore, $\left\langle x^{2 l}, U_{2 n}\right\rangle=0$ for $0 \leqslant l \leqslant n-1$ and $\left\langle x^{2 n}, U_{2 n}\right\rangle=(\sqrt{2})^{2 n+2} \gamma_{2 n+1}$. Similarly, $\left\langle x^{2 l+1}, U_{2 n+1}\right\rangle=0$ for $0 \leqslant l \leqslant n-1$ and $\left\langle x^{2 n+1}, U_{2 n+1}\right\rangle=(\sqrt{2})^{2 n+3} \gamma_{2 n+2}$. Therefore,

$$
\left\langle U_{2 n}, U_{2 n}\right\rangle=2 \gamma_{2 n+1} \text { and }\left\langle U_{2 n+1}, U_{2 n+1}\right\rangle=2 \gamma_{2 n+2} \text {. }
$$

This completes the proof of the lemma.

Now we complete the proof of $(29)$. For $f, g \in C_{b}(\mathbb{R})$, if

$$
\begin{aligned}
& f_{k}=\frac{1}{4 \pi} \int_{-2 \sqrt{2}}^{2 \sqrt{2}} f(x) U_{k}(x) \sqrt{8-x^{2}} d x, \\
& g_{k}=\frac{1}{4 \pi} \int_{-2 \sqrt{2}}^{2 \sqrt{2}} g(x) U_{k}(x) \sqrt{8-x^{2}} d x,
\end{aligned}
$$

then

$$
\langle f, g\rangle=\sum_{k=0}^{\infty} f_{k} g_{k} 2 \gamma_{k+1}
$$




$$
\begin{gathered}
=\frac{1}{8 \pi^{3}} \int_{-2 \sqrt{2}}^{2 \sqrt{2}} \int_{-2 \sqrt{2}}^{2 \sqrt{2}} f(x) g(y) \sqrt{8-x^{2}} \sqrt{8-y^{2}} \\
\times\left[\pi \sum_{k=0}^{\infty} U_{k}(x) U_{k}(y) \gamma_{k+1}\right] d x d y \\
=\frac{1}{8 \pi^{3}} \int_{-2 \sqrt{2}}^{2 \sqrt{2}} \int_{-2 \sqrt{2}}^{2 \sqrt{2}} f(x) g(y) \sqrt{8-x^{2}} \sqrt{8-y^{2}} F(x, y) d x d y,
\end{gathered}
$$

where

$$
\begin{aligned}
F(x, y) & =\pi \sum_{k=0}^{\infty} U_{k}(x) U_{k}(y) \gamma_{k+1} \\
& =2 \int_{-\infty}^{\infty} \frac{z-z^{3}}{2\left(1-z^{2}\right)^{2}+z^{2}\left(x^{2}+y^{2}\right)-z\left(1+z^{2}\right) x y} d s
\end{aligned}
$$

with $z=(\sin s) / s$. Now (63) holds due to (62) and orthogonality of Chebyshev polynomial with respect to the Wigner semicircular law, that is,

$$
\int_{-2 \sqrt{2}}^{2 \sqrt{2}} U_{n}(x) U_{m}(x) \frac{1}{4 \pi} \sqrt{8-x^{2}} d x=\delta_{m n} .
$$

And (64) is a straightforward consequence of the Fourier analysis using the following fact

$$
U_{n}(x)=\frac{\sin [(n+1) \theta]}{\sin \theta}, \quad x=2 \sqrt{2} \cos \theta .
$$

This completes the proof of (29).

Recent development. Recently, after submission of our paper, M. Shcherbina [16] improved our result by removing the restriction $b_{n} \gg \sqrt{n}$ and proved it for all $b_{n}$ which satisfies $b_{n} \rightarrow \infty$ and $b_{n} / n \rightarrow 0$ as $n \rightarrow \infty$.

Acknowledgment. We thank the referee for her/his constructive comments which have led to a significant improvement in presentation.

5. Some MATLAB simulation results. Here is what we found in MATLAB simulations.

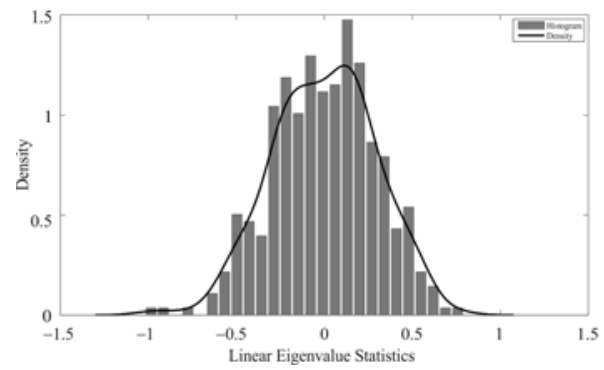

a) $n=2000, b_{n}=n^{0.2}$. Fourth moment $/(\text { variance })^{2}=2.92$.

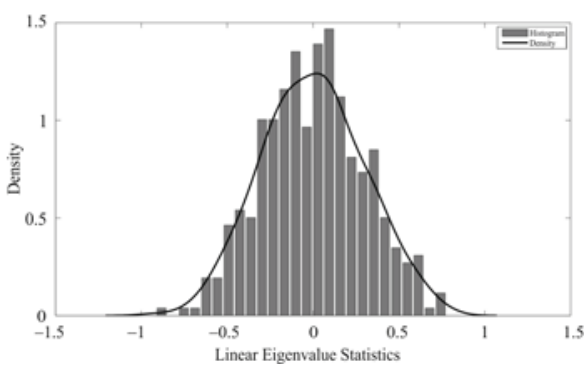

b) $n=2000, b_{n}=n^{0.4}$. Fourth moment $/(\text { variance })^{2}=2.71$. 

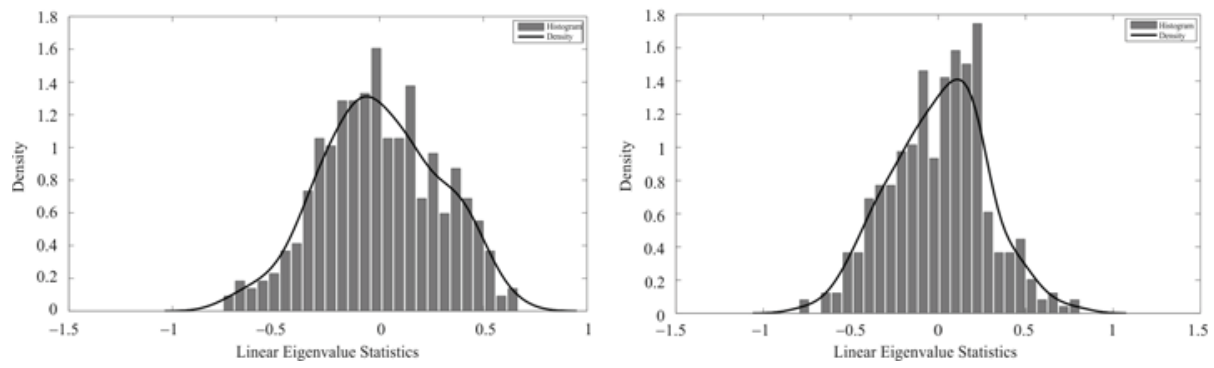

c) $n=2000, b_{n}=n^{0.6}$. Fourth moment $/(\text { variance })^{2}=2.57$.
d) $n=2000, b_{n}=n^{0.8}$. Fourth moment $/(\text { variance })^{2}=2.91$.

Fig. 3. The eigenvalue statistics was sampled 400 times. The test function was

$$
\phi(x)=\sqrt{16-x^{2}} .
$$

In the following example we had taken a different test function.

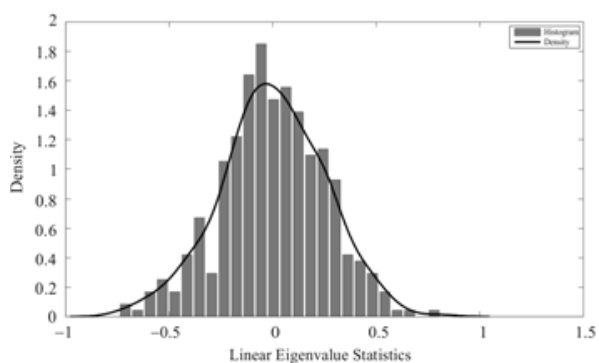

a) $n=2000, b_{n}=n^{0.2}$. Fourth moment $/(\text { variance })^{2}=3.08$.

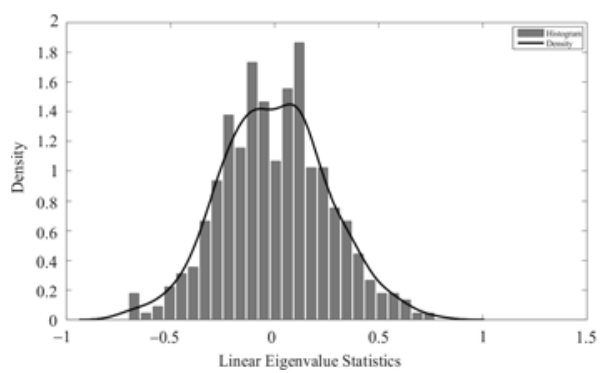

c) $n=2000, b_{n}=n^{0.6}$. Fourth moment $/(\text { variance })^{2}=3.00$.

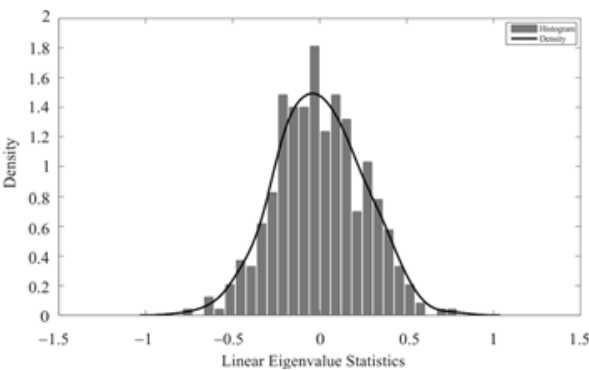

b) $n=2000, b_{n}=n^{0.4}$. Fourth moment $/(\text { variance })^{2}=2.94$.

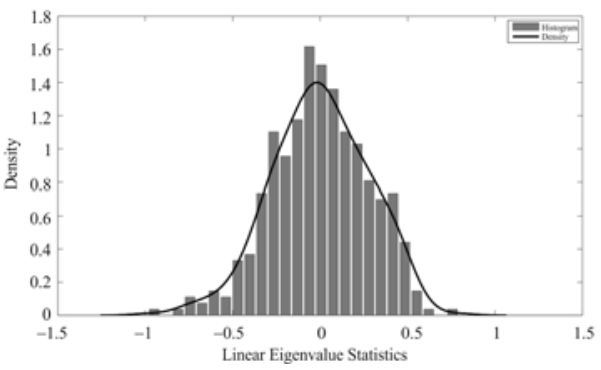

d) $n=2000, b_{n}=n^{0.8}$. Fourth moment $/(\text { variance })^{2}=3.08$.

Fig. 4. The eigenvalue statistics was sampled 400 times. The test function was

$$
\phi(x)=e^{-x^{2}} .
$$

\section{REFERENCES}

1. Anderson G.W., Guionnet A., Zeitouni O. An introduction to random matrices. Cambridge: Cambridge Univ. Press, 2010, 492 p.

2. Andrews G. E., Askey R., Roy R. Special functions. Encyclopedia of Mathematics and its Applications. Cambridge: Cambridge Univ. Press, 1999, 664 p. 
3. Casati G., Izrailev F., Molinari L. Scaling properties of the eigenvalue spacing distribution for band random matrices. - J. Phys. A: Mathematical and General, 1991, v. 24 , № 20 , p. 4755 .

4. Casati G., Molinari L., Izrailev F. Scaling properties of band random matrices. Phys. Rev. Lett., 1990, v. 64, № 16, p. 1851-1854.

5. Крамер Г. Математические методы статистики. М.: Мир, 1975, 648 с.

6. Dharmadhikari S., Fabian V., Jogdeo K. Bounds on the moments of martingales. Ann. Math. Statist., 1968, v. 39, №6, p. 1719-1723.

7. Эрдёu Л. Универсальность случайных матриц Вигнера: обзор последних результатов. - Успехи матем. наук., т. 66, № 3, с. 67-198.

8. Fyodorov Y. V., Mirlin A.D. Scaling properties of localization in random band matrices: a $\sigma$-model approach. - Phys. Rev. Lett., 1991, v. 67, № 18, p. 2405.

9. Li L., Soshnikov A. Central limit theorem for linear statistics of eigenvalues of band random matrices. - Random Matrices Theory Appl., 2013, v. 2, № 4, p. 50.

10. Lytova A., Pastur L. Central limit theorem for linear eigenvalue statistics of random matrices with independent entries. - Ann. Probab., 2009, v. 37, № 5, p. 1778-1840.

11. Mirlin A.D., Fyodorov Y.V., Dittes F.-M., Quezada J., Seligman T.H. Transition from localized to extended eigenstates in the ensemble of power-law random banded matrices. - Phys. Rev. E, 1996, v. 54, № 4, p. 3221.

12. Molchanov S.A., Pastur L.A., Khorunzhii A. Limiting eigenvalue distribution for band random matrices. - Theoret. Math. Phys., 1992, v. 90, № 2, p. 108-118.

13. O'Rourke S., Renfrew D., Soshnikov A. On fluctuations of matrix entries of regular functions of wigner matrices with non-identically distributed entries. - J. Theoret. Probab., 2013, v. 26, № 3, p. 750-780.

14. Pizzo A., Renfrew D., Soshnikov A. Fluctuations of matrix entries of regular functions of Wigner matrices. - J. Statist. Phys., 2012, v. 146, № 3, p. 550-591.

15. Shcherbina $M$. Central limit theorem for linear eigenvalue statistics of the wigner and sample covariance random matrices. - J. Math. Phys. Anal. Geom., 2011, v. 7, № 2, p. $176-192$.

16. Shcherbina M. On fluctuations of eigenvalues of random band matrices. - J. Stat. Phys., 2015, v. 4, № 1, p. 73-90.

17. Tulino A.M., Verdú S. Random Matrix Theory and Wireless Communications, Vol. 1. Princeton: Now Publishers Inc., 2004, 182 p.

18. Verdú S., Shamai S. Spectral efficiency of CDMA with random spreading. - IEEE Trans. Inform. Theory, 1999, v. 45, № 2, p. 622-640.

19. Wigner E. P. Characteristic vectors of bordered matrices with infinite dimensions. Ann. Math., 1955, v. 62, № 3, p. 548-564.

20. Wigner E.P. Characteristics vectors of bordered matrices with infinite dimensions. II. - Ann. Math., 1957, v. 65, № 2, p. 203-207.

21. Wishart $J$. The generalised product moment distribution in samples from a normal multivariate population. - Biometrika, 1928, v. 20A, № 1-2, p. 32-52.

Поступила в редакцию

4.II.2015 